\title{
Frames and numerical approximation II: generalized sampling
}

\author{
Ben Adcock \\ Simon Fraser University, Canada \\ Daan Huybrechs \\ KU Leuven, Belgium \\ Report TW689, February 2019
}

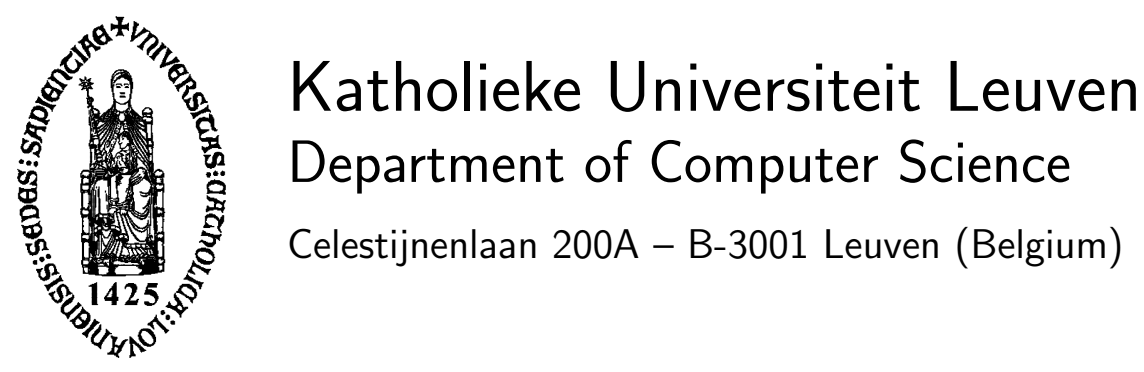




\title{
Frames and numerical approximation II: generalized sampling
}

\author{
Ben Adcock \\ Simon Fraser University, Canada \\ Daan Huybrechs \\ KU Leuven, Belgium \\ Report TW689, February 2019 \\ Department of Computer Science, KU Leuven
}

\begin{abstract}
In a previous paper "Frames and numerical approximation" we described the numerical properties of function approximation using frames, i.e. complete systems that are generally redundant but provide infinite representations with coefficients of bounded norm. Frames offer enormous flexibility compared to bases. We showed that, in spite of extreme ill-conditioning, a regularized projection onto a finite truncated frame can provide accuracy up to order $\sqrt{\epsilon}$, where $\epsilon$ is an arbitrarily small threshold. Here, we generalize the setting in two ways. First, we assume information or samples from $f$ from a wide class of linear operators acting on $f$, rather than inner products with the frame elements. Second, we allow oversampling, leading to least-squares approximations. The first property enables the analysis of fully discrete approximations based, for instance, on function values only. We show that the second property, oversampling, crucially leads to much improved accuracy on the order of $\epsilon$ rather than $\sqrt{\epsilon}$. Overall, we demonstrate that numerical function approximation using truncated frames leads to highly accurate approximations in spite of having to solve ill-conditioned systems of equations. Once the approximations start to converge, i.e. once sufficiently many degrees of freedom are used, any function $f$ can be approximated to within order $\epsilon$ with coefficients of small norm.
\end{abstract}

Keywords : function approximation, frames, function sampling, least squares AMS(MOS) Classification : Primary : 42C15, Secondary : 42C30, 41A10, $65 \mathrm{~T} 40$. 


\title{
Frames and numerical approximation II: generalized sampling
}

\author{
Ben Adcock \\ Department of Mathematics \\ Simon Fraser University \\ Canada
}

\author{
Daan Huybrechs \\ Department of Computer Science \\ KU Leuven \\ Belgium
}

February 19, 2019

\begin{abstract}
In a previous paper [4] we described the numerical properties of function approximation using frames, i.e. complete systems that are generally redundant but provide infinite representations with coefficients of bounded norm. Frames offer enormous flexibility compared to bases. We showed that, in spite of extreme ill-conditioning, a regularized projection onto a finite truncated frame can provide accuracy up to order $\sqrt{\epsilon}$, where $\epsilon$ is an arbitrarily small threshold. Here, we generalize the setting in two ways. First, we assume information or samples from $f$ from a wide class of linear operators acting on $f$, rather than inner products with the frame elements. Second, we allow oversampling, leading to least-squares approximations. The first property enables the analysis of fully discrete approximations based, for instance, on function values only. We show that the second property, oversampling, crucially leads to much improved accuracy on the order of $\epsilon$ rather than $\sqrt{\epsilon}$. Overall, we demonstrate that numerical function approximation using truncated frames leads to highly accurate approximations in spite of having to solve ill-conditioned systems of equations. Once the approximations start to converge, i.e. once sufficiently many degrees of freedom are used, any function $f$ can be approximated to within order $\epsilon$ with coefficients of small norm.
\end{abstract}

\section{Introduction}

Frames of Hilbert spaces are generalizations of orthonormal (or Riesz) bases which allow linear dependency amongst the elements, but retain a notion of stability via a relaxed version of Parseval's identity. The former endows frames with flexibility that orthonormal bases do not have, allowing frames to be constructed for approximation problems where constructing orthogonal bases with good (i.e. rapidly convergent) approximation properties is difficult or infeasible. For example, a basis can be 'enriched' by adding a few functions that capture a singularity. Or a periodic Fourier basis can be augmented with a few polynomials to capture the possible non-periodicity of a function $f$ to be approximated. Moreover, a basis for $L^{2}(D)$ associated with a domain $D$, is a frame for $L^{2}(\Omega)$ on any subset $\Omega \subset D$. The embedding of a domain with complicated geometry $\Omega$ in a simple bounding box $D$ is an ingredient in several numerical methods in scientific computing, such as embedded/fictitious domain methods, immersed boundary methods and others $[7,13,18]$. The mathematical structure of frames is consequently a convenient concept to analyze such general function approximation schemes in a unified manner.

In this paper, we continue a line of investigation that commenced in [4] on numerical approximation of functions using frames, rather than orthogonal bases. In this previous work, we considered 
computing the best approximation in a finite subset of frame. Unfortunately, frames necessarily lead to ill-conditioned linear systems of equations. The main contribution of [4] was a detailed analysis of the accuracy and conditioning of method for frame approximation obtained through regularization of the aforementioned linear system.

The main contributions of this paper are twofold. First, we show how to overcome a major issue with the method introduced in [4]: namely, that it can achieve at best $\mathcal{O}(\sqrt{\epsilon})$ accuracy, where $\epsilon$ is the regularization parameter used. We show how a modification of this method - based on oversampling - can achieve $\mathcal{O}(\epsilon)$ accuracy. Second, we extend the method of [4] to consider data (samples of the function to recover) that may be 'indirect', i.e. not inner products with the frame elements. This allows us to compute frame approximations from pointwise samples, for instance, which is commonplace in practice, or from inner products with respect to another frame or basis. The latter is reminiscent of Petrov-Galerkin methods in numerical PDEs.

\subsection{Frames and numerical approximation}

An indexed family $\Phi:=\left\{\phi_{n}\right\}_{n=1}^{\infty}$ is a frame for a Hilbert space $\mathrm{H}$ if it satisfies the frame condition

$$
A\|f\|^{2} \leq \sum_{n=1}^{\infty}\left|\left\langle f, \phi_{n}\right\rangle\right|^{2} \leq B\|f\|^{2}, \quad \forall f \in \mathrm{H},
$$

where $A, B>0$ are positive constants and $\|\cdot\|$ is the norm on $\mathrm{H}$.

The main concern of [4] was the computation of the best approximation, i.e. the orthogonal projection, in the truncated space $\mathrm{H}_{N}:=\operatorname{span}\left(\Phi_{N}\right)$ spanned by the first $N$ frame elements $\Phi_{N}:=$ $\left\{\phi_{n}\right\}_{n=1}^{N}$. This approximation is given by $\mathcal{P}_{N} f=\sum_{n=1}^{N} x_{n} \phi_{n}$, where $\boldsymbol{x}=\left(x_{n}\right)_{n=1}^{N}$ is a solution of the linear system

$$
\boldsymbol{G}_{N} \boldsymbol{x}=\boldsymbol{y},
$$

where $\boldsymbol{y}=\left\{\left\langle f, \phi_{n}\right\rangle\right\}_{n=1}^{N}$ and $\boldsymbol{G}_{N}=\left\{\left\langle\phi_{n}, \phi_{m}\right\rangle\right\}_{m, n=1}^{N}$ is the Gram matrix of $\Phi_{N}$.

Unfortunately, as shown in [4] the matrix $\boldsymbol{G}_{N}$ is necessary ill-conditioned for large $N$. Moreover, the coefficients $\boldsymbol{x}$ can also grow arbitrarily large, making them impossible to compute in floating point arithmetic for sufficiently large $N$. The remedy proposed in [4] was to regularize (1.2) by using a truncated Singular Value Decomposition (SVD) of $\boldsymbol{G}_{N}$ with a threshold parameter $\epsilon>0$ below which all the singular values are discarded. This results in a new projection $\mathcal{P}_{N}^{\epsilon} f=\sum_{n=1}^{N}\left(\boldsymbol{x}^{\epsilon}\right)_{n} \phi_{n}$, where $\boldsymbol{x}^{\epsilon}$ is the regularized solution of (1.2). To make the distinction clear, this is referred to as the numerical frame approximation, as opposed to the best frame approximation (i.e. the exact orthogonal projection, which generally cannot be computed).

The main result of [4] is the following:

Theorem 1.1 ([4]). The truncated $S V D$ projection $\mathcal{P}_{N}^{\epsilon}$ satisfies

$$
\left\|f-\mathcal{P}_{N}^{\epsilon} f\right\| \leq\left\|f-\sum_{n=1}^{N} z_{n} \phi_{n}\right\|+\sqrt{\epsilon}\|\boldsymbol{z}\|, \quad \forall \boldsymbol{z}=\left\{z_{n}\right\}_{n=1}^{N} \in \mathbb{C}^{N}, f \in \mathrm{H} .
$$

Moreover, the (absolute) condition number of the mapping $\boldsymbol{y} \mapsto \mathcal{P}_{N}^{\epsilon} f$ is at most $1 / \sqrt{\epsilon}$.

Observe that the right hand side in (1.3) contains two terms. Theorem 1.1 states that the regularized projection behaves like the best approximation to $f$ in the span of $\Phi_{N}$ (the first term), as long as the coefficients have sufficiently small norm (second term). Furthermore, convergence can only be expected to an accuracy up to the order of $\sqrt{\epsilon}$. Whether or not this accuracy is achieved, 
depends on the existence of a representation $\sum_{n=1}^{N} z_{n} \phi_{n}$ in the span of $\Phi_{N}$ with that accuracy and with small norm $\|\boldsymbol{z}\|$ of its coefficients. This question can be studied on a frame-by-frame basis, as done in [4] for a variety of examples.

Strictly speaking, Theorem 1.1 holds for any finite function set that gives rise to a well-defined Gram matrix. The main reason for restricting ourselves precisely to frames is the following. The frame condition guarantees that any function $f \in \mathrm{H}$ can be represented to any desired accuracy in the norm of $\mathrm{H}$, with coefficients whose norm is bounded up to a constant by the norm of $f$. Thus, eventually (i.e., for increasing $N$ ), all functions in $\mathrm{H}$ can be approximated to within $\sqrt{\epsilon}$ accuracy. Conversely, if $\Phi$ is not a frame, there must exist functions in $\mathrm{H}$ not well represented by any expansion in $\Phi_{N}$ with coefficients of bounded norm. By the second term in (1.3), one accordingly loses accuracy. However, even in that case, Theorem 1.1 might well be used to show convergence in a large subspace of $\mathrm{H}$.

\subsection{Main results}

In this paper, we generalize Theorem 1.1 in two ways. First, in $\S 3$ we develop a modified projection that overcomes the $\sqrt{\epsilon}$ barrier. This is done by oversampling. Specifically, we consider the rectangular Gram matrix

$$
\boldsymbol{G}_{M, N}=\left\{\left\langle\phi_{n}, \phi_{m}\right\rangle\right\}_{m, n=1}^{M, N} \in \mathbb{C}^{M \times N},
$$

where $M \geq N$ is to be determined. As we explain in $\S 3.3, \boldsymbol{G}_{M, N}$ is still ill-conditioned for large $N$, even when $M \gg N$. Hence, as before, we construct an approximation by singular value thresholding. This leads to a regularized approximation $\mathcal{P}_{M, N}^{\epsilon} f$ whose coefficients $\boldsymbol{x}^{\epsilon}$ are the regularized solution of the least-squares problem

$$
\boldsymbol{G}_{M, N} \boldsymbol{x} \approx \boldsymbol{y}, \quad \boldsymbol{y}=\left\{\left\langle f, \phi_{m}\right\rangle\right\}_{m=1}^{M} .
$$

Our first main result is the following generalization of Theorem 1.1:

Theorem 1.2. The truncated SVD projection $\mathcal{P}_{M, N}^{\epsilon} f$ satisfies

$$
\left\|f-\mathcal{P}_{M, N}^{\epsilon} f\right\| \leq\left(1+\sqrt{B} \kappa_{M, N}^{\epsilon}\right)\left\|f-\sum_{n=1}^{N} z_{n} \phi_{n}\right\|+\epsilon \lambda_{M, N}^{\epsilon}\|\boldsymbol{z}\|, \quad \forall \boldsymbol{z} \in \mathbb{C}^{N}
$$

for certain constants $\kappa_{M, N}^{\epsilon}, \lambda_{M, N}^{\epsilon}>0$, where $B$ is the upper frame bound of (1.1). The (absolute) condition number of the mapping $\boldsymbol{y} \mapsto \mathcal{P}_{M, N}^{\epsilon} f$ is precisely $\kappa_{M, N}^{\epsilon}$. Moreover, these constants satisfy

$$
\kappa_{M, N}^{\epsilon} \leq \frac{1}{\sqrt{\epsilon}}, \quad \lambda_{M, N}^{\epsilon} \leq \frac{1}{\sqrt{\epsilon}}
$$

and, for fixed $N$,

$$
\limsup _{M \rightarrow \infty} \kappa_{M, N}^{\epsilon} \leq \frac{1}{\sqrt{A}}, \quad \limsup _{M \rightarrow \infty} \lambda_{M, N}^{\epsilon} \leq \frac{1}{\sqrt{A}}
$$

Hence, for sufficiently large $M$, the error is within a constant factor of (1.3), except that $\sqrt{\epsilon}$ has, crucially, been replaced by $\epsilon$. In this paper, we quantify this requisite rate of growth of $M$ with $N$ in terms of the stable sampling rate. The question 'how large is sufficiently large' depends completely on the frame under consideration, and thus must be analyzed on a case-by-case basis. In $\S 5$, we illustrate an example frame for which the stable sampling rate is provably linear, i.e. there exists a $C \geq 1$ such that setting $M \geq C N$ implies that the constants $\kappa_{M, N}^{\epsilon}$ and $\lambda_{M, N}^{\epsilon}$ are bounded independently of $\epsilon$. Alternatively, this rate can also be computed numerically, as we explain in $\S 4.5$. 
Our second main contribution, introduced in $\S 4$, considers a generalization of this setup. Instead of inner products, the 'data' about the function $f$ is now given by bounded linear functionals $\ell_{m, M}: \mathrm{G} \rightarrow \mathbb{C}, m=1, \ldots, M$, which may depend on $M$ and which may be only defined on a dense subspace $\mathrm{G}$ of $\mathrm{H}$ (e.g. in the case of pointwise evaluations when $H=\mathrm{L}^{2}(\Omega)$ we consider $\mathrm{G}=\mathrm{L}^{\infty}(\Omega)$ ). Very much reminiscent of the frame condition (1.1), we assume this data is sufficiently 'rich' so as to stably recover $f$. Specifically, we assume that

$$
A^{\prime}\|f\|^{2} \leq \liminf _{M \rightarrow \infty} \sum_{m=1}^{M}\left|\ell_{m, M}(f)\right|^{2} \leq \limsup _{M \rightarrow \infty} \sum_{m=1}^{M}\left|\ell_{m, M}(f)\right|^{2} \leq B^{\prime}\|f\|^{2}, \quad \forall f \in \mathrm{G},
$$

for constants $A^{\prime}, B^{\prime}>0$.

As in the previous setting, we construct an approximation from the data $\boldsymbol{y}=\left\{\ell_{m, M}(f)\right\}_{m=1}^{M}$ via regularization of the least-squares problem

$$
\boldsymbol{G}_{M, N} \boldsymbol{x} \approx \boldsymbol{y}, \quad \boldsymbol{y}=\left\{\ell_{m, M}(f)\right\}_{m=1}^{M},
$$

where $\boldsymbol{G}_{M, N}=\left\{\ell_{m, M}\left(\phi_{n}\right)\right\}_{m, n=1}^{M, N}$. Our main result for this setup is the following:

Theorem 1.3. The truncated SVD projection $\mathcal{P}_{M, N}^{\epsilon} f$ satisfies

$$
\left\|f-\mathcal{P}_{M, N}^{\epsilon} f\right\| \leq\left\|f-\sum_{n=1}^{N} z_{n} \phi_{n}\right\|+\kappa_{M, N}^{\epsilon}\left\|f-\sum_{n=1}^{N} z_{n} \phi_{n}\right\|_{M}+\epsilon \lambda_{M, N}^{\epsilon}\|\boldsymbol{z}\|, \quad \forall \boldsymbol{z} \in \mathbb{C}^{N},
$$

for constants $\kappa_{M, N}^{\epsilon}, \lambda_{M, N}^{\epsilon}>0$. The (absolute) condition number of the mapping $\boldsymbol{y} \mapsto \mathcal{P}_{M, N}^{\epsilon} f$ is precisely $\kappa_{M, N}^{\epsilon}$. Moreover, these constants satisfy

$$
\kappa_{M, N}^{\epsilon} \leq \frac{\sqrt{B}}{\epsilon}, \quad \lambda_{M, N}^{\epsilon} \leq \frac{\sqrt{B}}{\epsilon}
$$

and, for fixed $N$,

$$
\limsup _{M \rightarrow \infty} \kappa_{M, N}^{\epsilon} \leq \frac{1}{\sqrt{A^{\prime}}}, \quad \limsup _{M \rightarrow \infty} \lambda_{M, N}^{\epsilon} \leq \frac{1}{\sqrt{A^{\prime}}}
$$

Here, $\|g\|_{M}^{2}=\sum_{m=1}^{M}\left|\ell_{m, M}(g)\right|^{2}$ is the discrete semi-norm defined by the data. Note that the bound (1.6) in Theorem 1.2 is strictly a special case of (1.9), corresponding to $\mathrm{G}=\mathrm{H}$ and $\ell_{m, M}(f)=\left\langle f, \phi_{m}\right\rangle, m=1, \ldots, M$ (in this case, one has $\|\cdot\|_{M} \leq \sqrt{B}\|\cdot\|$ due to the frame condition).

As in Theorem 1.2, this result yields an error bound depending on $f-\sum_{n=1}^{N} z_{n} \phi_{n}$ (measured in some norm) and $\epsilon\|\boldsymbol{z}\|$ with constants that are $\mathcal{O}(1)$ for sufficiently large $M$. The question of how large $M$ must be depends on the frame, and in this case, on the linear functionals $\ell_{m, M}$. As before, in $\S 5$ we illustrate an example where $M \geq C N$ suffices. Also as before, the constants can be computed numerically (see $\S 4.5$ ).

\subsection{Relation to other work}

As discussed, this paper is a continuation of [4], in which the systematic study of numerical frame approximation was commenced. This study had its origins on earlier work on so-called Fourier extensions $[5,12]$, which are particular frames arising as restrictions of the Fourier basis on a box to a subdomain.

Our use of oversampling here is inspired by earlier work on generalized sampling in Hilbert spaces by the first author and Hansen $[1,2,3]$. That work considered both sampling and approximation 
using orthonormal bases and frames, introducing the stable sampling rate as well, but did not address the ill-conditioning issue for approximation in the latter. Note that the tall rectangular matrix (1.4) is an uneven section of the infinite Gram matrix of the full frame, whereas the matrix $\boldsymbol{G}_{N}$ of (1.2) is a finite section. Using uneven as opposed to finite sections is a well-known trick in computational spectral theory $[10,11,14]$.

For a more in-depth discussion of relations between this work and standard frame theory, we refer to [4].

Our focus in this paper is accuracy and conditioning of the regularized frame approximations. We do not consider efficiency, i.e. computational time, which is very much dependent on the particular frame under consideration. We note in passing that there are efficient numerical methods for solving (1.5) and (1.8) for certain frames [15, 16, 17]. A description of these algorithms at the generic level will be presented in a future work.

\section{Preliminaries}

Our main results are contained in $§ 3-5$. First, however, we give some necessary background on frames and frame theory.

\subsection{Bases and frames}

For the remainder of this paper, $\Phi=\left\{\phi_{n}\right\}_{n \in I}$ is an indexed family in a separable Hilbert space $\mathrm{H}$ over the field $\mathbb{C}$, where $I$ is a countable index set (for convenience and generality, we now allow $I$ to be an abstract index set, rather than $\mathbb{N}$ as in $\S 1$ ). We write $\langle\cdot, \cdot\rangle$ and $\|\cdot\|$ for the inner product and norm on $\mathrm{H}$ respectively.

Definition 2.1. An indexed family $\Phi=\left\{\phi_{n}\right\}_{n \in I}$ is an orthonormal basis for $H$ if $\operatorname{span}(\Phi)$, the vector space of all finite linear combinations of elements of $\Phi$, is dense in $\mathrm{H}$ and $\left\langle\phi_{n}, \phi_{m}\right\rangle=\delta_{n, m}$, $\forall n, m \in I$.

Recall that orthonormal bases satisfy Parseval's identity

$$
\|f\|^{2}=\sum_{n \in I}\left|\left\langle f, \phi_{n}\right\rangle\right|^{2}, \quad \forall f \in \mathrm{H} .
$$

Equivalently,

$$
\|\boldsymbol{x}\|=\left\|\sum_{n \in I} x_{n} \phi_{n}\right\|, \quad \forall \boldsymbol{x}=\left\{x_{n}\right\}_{n \in I} \in \ell^{2}(I) .
$$

Here and throughout, $\ell^{2}(I)$ denotes the space of square-summable sequences indexed over $I$, and $\|\cdot\|$ denotes its norm, i.e. $\|\boldsymbol{x}\|=\sqrt{\sum_{n \in I}\left|x_{n}\right|^{2}}$.

Definition 2.2. An indexed family $\Phi=\left\{\phi_{n}\right\}_{n \in I}$ is a Riesz basis for $\mathrm{H}$ if $\operatorname{span}(\Phi)$ is dense in $\mathrm{H}$, and there exist constants $A, B>0$ such that

$$
A\|\boldsymbol{x}\|^{2} \leq\left\|\sum_{n \in I} x_{n} \phi_{n}\right\|^{2} \leq B\|\boldsymbol{x}\|^{2}, \quad \forall \boldsymbol{x}=\left\{x_{n}\right\}_{n \in I} \in \ell^{2}(I) .
$$

Note that (2.3) is a relaxed version of Parseval's identity (2.2). Throughout this paper, whenever constants $A$ and $B$ are introduced in an inequality such as this, they will be taken to be the optimal constants such that the inequality holds. 
Definition 2.3. An indexed family $\Phi=\left\{\phi_{n}\right\}_{n \in I}$ is a frame for $\mathrm{H}$ if

$$
A\|f\|^{2} \leq \sum_{n \in I}\left|\left\langle f, \phi_{n}\right\rangle\right|^{2} \leq B\|f\|^{2}, \quad \forall f \in \mathrm{H},
$$

for positive constants $A, B>0$. A frame is tight if $A=B$.

We refer to (2.4) as the frame condition. Note that it implies that $\Phi$ is dense in H. It follows from (2.3) that a Riesz basis is also a frame with the same constants $A, B$. [8, Prop. 3.6.4]. But a frame need not be a Riesz basis. In fact, frames are generally not $\omega$-independent (see $[8$, Sec. 6.1], for example): that is, there exist nonzero coefficients $\left\{x_{n}\right\}_{n \in I}$ for which the $\operatorname{sum} \sum_{n \in I} x_{n} \phi_{n}$ converges in $\mathrm{H}$ and satisfies $\sum_{n \in I} x_{n} \phi_{n}=0$. Conversely, bases are always $\omega$-independent. As mentioned, this redundancy gives frames far greater flexibility than bases, making them easier to construct for particular problems.

Definition 2.4. A frame $\Phi$ is exact if it ceases to be a frame when any one element is removed. Otherwise it is inexact.

Note that a frame is exact if and only if it is a Riesz basis [8, Theorem 5.5.4]. Hence, for the remainder of this paper we will assume that all frames are inexact.

Definition 2.5. A frame $\Phi=\left\{\phi_{n}\right\}_{n \in I}$ is linearly independent if every finite subset $\left\{\phi_{n}\right\}_{n \in J}$, $|J|<\infty$, is linearly independent.

We shall also assume that all frames are linearly independent. This is mainly for convenience, and it will be the case in the examples discussed. Note that a linearly-independent frame is not necessarily a Riesz basis, as $\omega$-independence for the infinite frame is a stronger condition. See [8, Chpt. 6] for further discussion.

\subsection{Operators on frames}

Associated to any frame $\Phi$ (and therefore any Riesz basis) is the so-called synthesis operator

$$
\mathcal{T}: \ell^{2}(I) \rightarrow \mathrm{H}, \quad \boldsymbol{y}=\left\{y_{n}\right\}_{n \in I} \mapsto \sum_{n \in I} y_{n} \phi_{n}
$$

Its adjoint, the analysis operator, is given by

$$
\mathcal{T}^{*}: \mathrm{H} \rightarrow \ell^{2}(I), \quad f \mapsto\left\{\left\langle f, \phi_{n}\right\rangle\right\}_{n \in I},
$$

and the composition $\mathcal{S}=\mathcal{T}^{*}$, known as the frame operator, is

$$
\mathcal{S}: \mathrm{H} \rightarrow \mathrm{H}, \quad f \mapsto \sum_{n \in I}\left\langle f, \phi_{n}\right\rangle \phi_{n} .
$$

This operator is self-adjoint, bounded, invertible and positive with

$$
A\|f\|^{2} \leq\langle\mathcal{S} f, f\rangle \leq B\|f\|^{2} .
$$

See [8, Lemma 5.1.5]. Note that this inequality is equivalent to the frame condition (2.4). Note also that $\mathcal{S}=\mathcal{I}$ is the identity operator for an orthonormal basis. Similarly, $\mathcal{S}=A \mathcal{I}$ for a tight frame. However, for a general Riesz basis or frame, $\mathcal{S} \neq \mathcal{I}$. 
The Gram operator of a frame is defined by $\mathcal{G}=\mathcal{T}^{*} \mathcal{T}$. That is,

$$
\mathcal{G}: \ell^{2}(I) \rightarrow \ell^{2}(I), \boldsymbol{x}=\left\{x_{n}\right\}_{n \in I} \mapsto\left\{\sum_{m \in I}\left\langle\phi_{m}, \phi_{n}\right\rangle x_{m}\right\}_{n \in I}
$$

While this is a bounded operator on $\ell^{2}(I)$, it is generally not invertible. We may also view $\mathcal{G}$ as the infinite matrix $\boldsymbol{G}=\left\{\left\langle\phi_{n}, \phi_{m}\right\rangle\right\}_{n, m \in I}$. Throughout this paper all infinite matrices are equivalent to bounded operators on $\ell^{2}(I)$. Note that $\mathcal{G}$ is the identity if and only if $\Phi$ is an orthonormal basis.

\subsection{Dual frames}

Definition 2.6. A frame $\Psi=\left\{\psi_{n}\right\}_{n \in I} \subseteq \mathrm{H}$ is a dual frame for a given frame $\Phi$ if

$$
f=\sum_{n \in I}\left\langle f, \psi_{n}\right\rangle \phi_{n}=\sum_{n \in I}\left\langle f, \phi_{n}\right\rangle \psi_{n}, \quad \forall f \in \mathrm{H}
$$

If a frame $\Phi$ is also a Riesz basis then it has a unique dual frame $\Psi$, which is also a Riesz basis. In this case, the pair $(\Phi, \Psi)$ is biorthogonal:

$$
\left\langle\phi_{n}, \psi_{m}\right\rangle=\delta_{n, m}, \quad n, m \in I .
$$

Note that an orthonormal basis is self-dual, i.e. $\Psi=\Phi$. Conversely, an inexact frame (see Definition 2.4) necessarily has more than one dual frame. Moreover, a frame and its duals are not biorthogonal.

Definition 2.7. Let $\Phi=\left\{\phi_{n}\right\}_{n \in I}$ be a frame. The canonical dual frame of $\Phi$ is the frame $\Psi=\left\{\mathcal{S}^{-1} \phi_{n}\right\}_{n \in I}$.

The canonical dual is a frame $\left[8\right.$, Lem. 5.1.5], and its frame bounds are $B^{-1}$ and $A^{-1}$ respectively. In this case, (2.7) reads

$$
f=\sum_{n \in I}\left\langle f, \mathcal{S}^{-1} \phi_{n}\right\rangle \phi_{n}=\sum_{n \in I}\left\langle\mathcal{S}^{-1} f, \phi_{n}\right\rangle \phi_{n}
$$

We refer to the coefficients $\boldsymbol{a}=\left\{\left\langle f, \mathcal{S}^{-1} \phi_{n}\right\rangle\right\}_{n \in I}$ as the frame coefficients of $f$. Note that these coefficients have the property that, amongst all possible representations of $f$ in $\Phi$, they have the smallest norm [8, Lem. 5.4.2]. Specifically, if $f=\sum_{n \in I} a_{n} \phi_{n}=\sum_{n \in I} c_{n} \phi_{n}$ for some $\boldsymbol{c}=\left\{c_{n}\right\}_{n \in I}$, then $\|\boldsymbol{c}\| \geq\|\boldsymbol{a}\|$.

\subsection{Truncations of frames}

Let $\Phi_{N}=\left\{\phi_{n}\right\}_{n \in I_{N}}$ be the truncated system, where $I_{N} \subseteq I$ is a finite index set with $\left|I_{N}\right|=N$. For convenience, we assume that the index sets $\left\{I_{N}\right\}_{N \in \mathbb{N}}$ satisfy

$$
I_{1} \subseteq I_{2} \subseteq \ldots, \quad \bigcup_{N=1}^{\infty} I_{N}=I .
$$

Let $\mathrm{H}_{N}=\operatorname{span}\left(\Phi_{N}\right)$ and note that $\Phi_{N}$ is a frame for $\mathrm{H}_{N}$. We write $A_{N}, B_{N}>0$ for the frame bounds:

$$
A_{N}\|f\|^{2} \leq \sum_{n \in I_{N}}\left|\left\langle f, \phi_{n}\right\rangle\right|^{2} \leq B_{N}\|f\|^{2}, \quad \forall f \in \mathrm{H}_{N} .
$$


We also let $\mathcal{T}_{N}: \mathbb{C}^{N} \rightarrow \mathrm{H}_{N}, \mathcal{T}_{N}^{*}: \mathrm{H}_{N} \rightarrow \mathbb{C}^{N}$ and $\mathcal{S}_{N}=\mathcal{T}_{N} \mathcal{T}_{N}^{*}: \mathrm{H}_{N} \rightarrow \mathrm{H}_{N}$ denote the truncated synthesis, analysis and frame operators respectively. The truncated Gram operator is $\mathcal{G}_{N}=\mathcal{T}_{N}^{*} \mathcal{T}_{N}$ : $\mathbb{C}^{N} \rightarrow \mathbb{C}^{N}$ and is equivalent to the $N \times N$ Gram matrix

$$
\boldsymbol{G}_{N}=\left\{\left\langle\phi_{m}, \phi_{n}\right\rangle\right\}_{n, m \in I_{N}} \in \mathbb{C}^{N \times N} .
$$

Since $\Phi$ is linearly independent, $\Phi_{N}$ is also a basis for $\mathrm{H}_{N}$. However, it is generally not well conditioned. The following lemma relates conditioning of $\boldsymbol{G}_{N}$ to the frame bounds $A_{N}, B_{N}$ :

Lemma 2.8 (Lemma 4 of [4]). The truncated Gram matrix $\boldsymbol{G}_{N}$ of a linearly-independent frame $\Phi$ is invertible with $\left\|\boldsymbol{G}_{N}^{-1}\right\|^{-1}=A_{N}$ and $\left\|\boldsymbol{G}_{N}\right\|=B_{N}$, where $A_{N}$ and $B_{N}$ are the frame bounds of the truncated frame $\Phi_{N}$. In particular, the condition number of $\boldsymbol{G}_{N}$ is precisely the ratio of the truncated frame bounds: $\kappa\left(\boldsymbol{G}_{N}\right)=\left\|\boldsymbol{G}_{N}\right\|\left\|\boldsymbol{G}_{N}^{-1}\right\|=B_{N} / A_{N}$.

While $\boldsymbol{G}_{N}$ is invertible, it is necessarily ill-conditioned for large $N$. Indeed:

Lemma 2.9 (Lemma 5 of [4]). Let $\Phi$ be a linearly-independent frame. Then

(i) the sequences $\left\{A_{N}\right\}_{N \in \mathbb{N}}$ and $\left\{B_{N}\right\}_{N \in \mathbb{N}}$ are monotonically nonincreasing and nondecreasing respectively,

(ii) $B_{N} \leq B$ for all $N$ and $B_{N} \rightarrow B$ as $N \rightarrow \infty$,

(iii) $\inf _{N} A_{N}>0$ if and only if $\Phi$ is a Riesz basis.

\section{Oversampling and order epsilon accuracy}

As noted, a limitation of the regularized projection of [4] for solving (1.2) is that it can at best achieve order $\sqrt{\epsilon}$ accuracy. In this section we present an alternative which attains order $\epsilon$ accuracy.

The approach is simple and is inspired by the ideas of generalized sampling [1,2]. Suppose we view the right-hand side $\boldsymbol{y}=\left\{\left\langle f, \phi_{m}\right\rangle\right\}_{m \in I_{M}}$ as data. Then, rather than trying to approximate $f$ in the space $\Phi_{M}$ of the same size, we seek to approximate in the smaller space $\Phi_{N}$, where $N \leq M$ is chosen suitably small to ensure accuracy and good conditioning of the approximation. Equivalently, if $N$ is fixed, we oversample: that is, we allow $M \geq N$. Note that for the time being, we continue to assume that the data consists of inner products with a set $\Phi_{M}$. Thus, the generalization is simply that $\Phi_{M}$ may be larger than $\Phi_{N}$.

Let

$$
\boldsymbol{G}_{M, N}=\mathcal{T}_{M}^{*} \mathcal{T}_{N}=\left\{\left\langle\phi_{n}, \phi_{m}\right\rangle\right\}_{m \in I_{M}, n \in I_{N}} \in \mathbb{C}^{M \times N},
$$

be the truncated Gram matrix corresponding to truncation parameters $M$ (number of rows) and $N$ (number of columns). Note that $\boldsymbol{G}_{M, N}$ is the leading $M \times N$ submatrix of the infinite matrix $\boldsymbol{G}$. Then we define the vector $\boldsymbol{x} \in \mathbb{C}^{N}$ as a solution of the least-squares problem

$$
\min _{\boldsymbol{z} \in \mathbb{C}^{N}}\left\|\boldsymbol{G}_{M, N} \boldsymbol{z}-\boldsymbol{y}\right\|, \quad \text { where } \boldsymbol{y}=\left\{\left\langle f, \phi_{m}\right\rangle\right\}_{m \in I_{M}}
$$

The following lemma shows that the problem (3.1) has a unique solution:

Lemma 3.1. For any $M$ and $N$ with $M \geq N$ we have $\mathcal{T}_{M}^{*} g=0$ for $g \in \mathrm{H}_{N}$ if and only if $g=0$. Equivalently, the matrix $\boldsymbol{G}_{M, N}$ has full rank. 
Proof. Let $g \in \mathrm{H}_{N}$ and write $g=\mathcal{T}_{N} \boldsymbol{x}$ for some $\boldsymbol{x} \in \mathbb{C}^{N}$. Observe that $\mathcal{T}_{M}^{*} g=\mathcal{T}_{M}^{*} \mathcal{T}_{N} \boldsymbol{x}=\boldsymbol{G}_{M, N} \boldsymbol{x}$. Since $\Phi$ is a linearly-independent frame, $g=0$ if and only if $\boldsymbol{x}=0$. Therefore $\boldsymbol{G}_{M, N}$ has full rank if and only if the statement

$$
\mathcal{T}_{M}^{*} g=0 \Longleftrightarrow g=0, \quad g \in \mathrm{H}_{N}
$$

holds. To show that it does we note that $B\|g\|^{2} \geq\left\|\mathcal{T}_{M}^{*} g\right\|^{2} \geq\left\|\mathcal{T}_{N}^{*} g\right\|^{2} \geq A_{N}\|g\|^{2}$, where in the last inequality we use the fact that $g \in \mathrm{H}_{N}$. This gives the result.

With this in hand, if $\boldsymbol{x}$ is the unique solution of (3.1), then we define the approximation

$$
f \approx \mathcal{P}_{M, N} f=\mathcal{T}_{N} \boldsymbol{x}
$$

Note that $\mathcal{P}_{M, N}: \mathrm{H} \rightarrow \mathrm{H}_{N}$ is the linear operator which takes $f$ to its approximation $\mathcal{T}_{N} \boldsymbol{x}$ with $\boldsymbol{x}$ the solution of (3.1).

Unfortunately, much like in the case without oversampling [4], the Gram matrix $\boldsymbol{G}_{M, N}$ is still ill-conditioned even if $M \gg N$ (see $\S 3.3$ ). Hence we need to regularize. Suppose that $\boldsymbol{G}_{M, N}$ has singular value decomposition

$$
\boldsymbol{G}_{M, N}=\boldsymbol{U} \boldsymbol{\Sigma} \boldsymbol{V}^{*}
$$

Let $\epsilon>0$ be fixed. Then we set

$$
\boldsymbol{x}^{\epsilon}=\left(\boldsymbol{G}_{M, N}^{\epsilon}\right)^{\dagger} y=\boldsymbol{V}\left(\boldsymbol{\Sigma}^{\epsilon}\right)^{\dagger} \boldsymbol{U}^{*} y
$$

where $\dagger$ denotes the pseudoinverse and $\boldsymbol{\Sigma}^{\epsilon}$ is the diagonal matrix with $n^{\text {th }}$ entry $\sigma_{n}$ if

$$
\sigma_{n}>\epsilon
$$

and zero otherwise. The corresponding approximation to $f$ is

$$
f \approx \mathcal{P}_{M, N}^{\epsilon} f=\mathcal{T}_{N} \boldsymbol{x}^{\epsilon}
$$

For convenience, we now define the mappings

$$
\mathcal{L}_{M, N}: \mathbb{C}^{M} \rightarrow \mathrm{H}_{N}, \boldsymbol{y} \mapsto \mathcal{T}_{N}\left(\boldsymbol{G}_{M, N}\right)^{\dagger} \boldsymbol{y}, \quad \mathcal{L}_{M, N}^{\epsilon}: \mathbb{C}^{M} \rightarrow \mathrm{H}_{N}, \boldsymbol{y} \mapsto \mathcal{T}_{N}\left(\boldsymbol{G}_{M, N}^{\epsilon}\right)^{\dagger} \boldsymbol{y}
$$

These map the data $\boldsymbol{y}$ to the corresponding approximations in $\mathrm{H}_{N}$. In particular, we have

$$
\mathcal{P}_{M, N}=\mathcal{L}_{M, N} \mathcal{T}_{M}^{*}, \quad \mathcal{P}_{M, N}^{\epsilon}=\mathcal{L}_{M, N}^{\epsilon} \mathcal{T}_{M}^{*}
$$

Note that the square Gram matrix $\boldsymbol{G}_{N}$ of $\S 1$ is precisely $\boldsymbol{G}_{N, N}$. Since $\boldsymbol{G}_{N}$ is invertible, it follows that the projections $\mathcal{P}_{N}^{\epsilon}$ and $\mathcal{P}_{N}$ discussed in [4] correspond exactly to the case $M=N$.

\subsection{Theoretical results}

We first introduce the following constants:

$$
\kappa_{M, N}^{\epsilon}=\max _{\substack{\boldsymbol{y} \in \mathbb{C}^{M} \\\|\boldsymbol{y}\|=1}}\left\|\mathcal{L}_{M, N}^{\epsilon} \boldsymbol{y}\right\|, \quad \lambda_{M, N}^{\epsilon}=\epsilon^{-1} \max _{\substack{\boldsymbol{z} \in \mathbb{C}^{N} \\\|\boldsymbol{z}\|=1}}\left\|\mathcal{T}_{N} \boldsymbol{z}-\mathcal{P}_{M, N}^{\epsilon} \mathcal{T}_{N} \boldsymbol{z}\right\|
$$

Note that $\kappa_{M, N}^{\epsilon}$ is precisely the operator norm of $\mathcal{L}_{M, N}^{\epsilon}: \mathbb{C}^{M} \rightarrow$ H. Since $\mathcal{L}_{M, N}^{\epsilon}$ is linear, it is also its absolute condition number, i.e. $\kappa_{M, N}^{\epsilon}$ measures the absolute effect of perturbations in the data $\boldsymbol{y}$ on the final approximation. Conversely, $\lambda_{M, N}^{\epsilon}$ measures how close $\mathcal{P}_{M, N}^{\epsilon}$ is to being a projection 
on the subspace $\mathrm{H}_{N}=\mathcal{T}_{N}\left(\mathbb{C}^{N}\right)$. Observe that $\lambda_{M, N}^{0}=0$, since $\mathcal{P}_{M, N}$ is a projection onto $\mathrm{H}_{N}$. For convenience, we now also define

$$
C_{M, N}^{\epsilon}=\sqrt{A} \max \left\{\kappa_{M, N}^{\epsilon}, \lambda_{M, N}^{\epsilon}\right\}
$$

The reason for this scaling will become clear in the next subsection.

Our first result concerns the approximation error of $\mathcal{P}_{M, N}^{\epsilon} f$ :

Theorem 3.2. The truncated $S V D$ approximation $\mathcal{P}_{M, N}^{\epsilon} f$ satisfies

$$
\left\|f-\mathcal{P}_{M, N}^{\epsilon} f\right\| \leq\left(1+\sqrt{B} \kappa_{M, N}^{\epsilon}\right)\left\|f-\mathcal{T}_{N} \boldsymbol{z}\right\|+\epsilon \lambda_{M, N}^{\epsilon}\|\boldsymbol{z}\|, \quad \forall \boldsymbol{z} \in \mathbb{C}^{N}
$$

In particular,

$$
\left\|f-\mathcal{P}_{M, N}^{\epsilon} f\right\| \leq \max \left\{1+\sqrt{\frac{B}{A}} C_{M, N}^{\epsilon}, \frac{1}{\sqrt{A}} C_{M, N}^{\epsilon}\right\} E_{N}^{\epsilon}(f),
$$

where $C_{M, N}^{\epsilon}$ is as in (3.6) and

$$
E_{N}^{\epsilon}(f)=\inf \left\{\left\|f-\mathcal{T}_{N} \boldsymbol{z}\right\|+\epsilon\|\boldsymbol{z}\|: \boldsymbol{z} \in \mathbb{C}^{N}\right\} .
$$

For the coefficients $\boldsymbol{x}^{\epsilon}$, we have the following:

Theorem 3.3. The coefficients $\boldsymbol{x}^{\epsilon}$ of the truncated $S V D$ projection $\mathcal{P}_{M, N}^{\epsilon}$ satisfy

$$
\left\|\boldsymbol{x}^{\epsilon}\right\| \leq \frac{\sqrt{B}}{\epsilon}\left\|f-\mathcal{T}_{N} \boldsymbol{z}\right\|+\|\boldsymbol{z}\|, \quad \forall z \in \mathbb{C}^{N} .
$$

Moreover, if $\boldsymbol{a}_{M, N}^{\epsilon} \in \ell^{2}(I)$ is the extension of $\boldsymbol{x}^{\epsilon}$ by zero, then

$$
\left\|\boldsymbol{a}-\boldsymbol{a}_{M, N}^{\epsilon}\right\| \leq\left(1+\frac{B}{\epsilon}\right) \sqrt{\sum_{n \in I \backslash I_{N}}\left|a_{n}\right|^{2}}+\epsilon \frac{\lambda_{M, N}^{\epsilon}}{\sqrt{A}}\|\boldsymbol{a}\|,
$$

where $\boldsymbol{a}=\left\{\left\langle f, \mathcal{S}^{-1} \phi_{n}\right\rangle\right\}_{n \in I}$ are the frame coefficients of $f \in \mathrm{H}$.

These results are corollaries of two more general theorems we present in $\S 4$ (see $\S 4.3$ for proofs). In order to interpret them, we first need to understand the behaviour of the constants $\kappa_{M, N}^{\epsilon}$ and $\lambda_{M, N}^{\epsilon}$ as $M \rightarrow \infty$. We discuss this next.

\subsection{The stable sampling rate and $\mathcal{O}(\epsilon)$ accuracy}

We have:

Proposition 3.4. The constants $\kappa_{M, N}^{\epsilon}$ and $\lambda_{M, N}^{\epsilon}$ satisfy

$$
\kappa_{M, N}^{\epsilon} \leq \frac{1}{\sqrt{\epsilon}}, \quad \lambda_{M, N}^{\epsilon} \leq \frac{1}{\sqrt{\epsilon}}, \quad \forall M, N \in \mathbb{N}, M \geq N .
$$

Moreover, for fixed $N$, $\lim \sup _{M \rightarrow \infty} \kappa_{M, N}^{\epsilon} \leq \frac{1}{\sqrt{A}}$ and $\limsup _{M \rightarrow \infty} \lambda_{M, N}^{\epsilon} \leq \frac{1}{\sqrt{A}}$, and therefore

$$
\limsup _{M \rightarrow \infty} C_{M, N}^{\epsilon} \leq 1 .
$$


See $\S 4.4$ for a proof. Motivated by this proposition, we now introduce the following concept:

Definition 3.5. Let $C_{M, N}^{\epsilon}$ be as in (3.6). For $1<\theta<\infty$ and $N \in \mathbb{N}$, the stable sampling rate is

$$
\Theta^{\epsilon}(N, \theta)=\min \left\{M: M \geq N, C_{M, N}^{\epsilon} \leq \theta\right\}
$$

For a given $N$, suppose that $M \geq \Theta^{\epsilon}(N, \theta)$. Then Theorem 3.2 gives that

$$
\left\|f-\mathcal{P}_{M, N}^{\epsilon} f\right\| \leq\left(1+\sqrt{\frac{B}{A}} \theta\right)\left\|f-\mathcal{T}_{N} \boldsymbol{z}\right\|+\epsilon \frac{\theta}{\sqrt{A}}\|\boldsymbol{z}\|, \quad \forall \boldsymbol{z} \in \mathbb{C}^{N} .
$$

Crucially, the error bound scales with $\epsilon$ as opposed to $\sqrt{\epsilon}$. In particular, this means that the approximation achieves order $\epsilon$ accuracy in the limit as $N \rightarrow \infty$, as opposed to $\sqrt{\epsilon}$ accuracy of the approximation $\mathcal{P}_{N}^{\epsilon} f$ (recall Theorem 1.1):

Corollary 3.6. For each $1<\theta<\infty$ the truncated $S V D$ approximation $\mathcal{P}_{M, N}^{\epsilon} f$ satisfies

$$
\limsup _{\substack{M, N \rightarrow \infty \\ M \geq \Theta^{\epsilon}(N, \theta)}}\left\|f-\mathcal{P}_{M, N}^{\epsilon} f\right\| \leq \epsilon \frac{\theta}{A}\|f\|,
$$

Moreover, the coefficients $\boldsymbol{x}^{\epsilon}$ satisfy

$$
\limsup _{\substack{M, N \rightarrow \infty \\ M \geq \Theta^{\epsilon}(N, \theta)}}\left\|\boldsymbol{x}^{\epsilon}\right\| \leq \frac{1}{\sqrt{A}}\|f\|, \quad \limsup _{\substack{M, N \rightarrow \infty \\ M \geq \Theta^{\epsilon}(N, \theta)}}\left\|\boldsymbol{a}-\boldsymbol{a}_{M, N}^{\epsilon}\right\| \leq \epsilon \frac{\theta}{A}\|\boldsymbol{a}\| .
$$

Proof. The proof is based on the frame coefficients $\boldsymbol{a}=\left\{\left\langle f, \mathcal{S}^{-1} \phi_{n}\right\rangle\right\}_{n \in I}$. Let $\boldsymbol{z}=\left\{a_{n}\right\}_{n \in I_{N}}$ in (3.12). Then $\|\boldsymbol{z}\| \leq\|\boldsymbol{a}\| \leq 1 / \sqrt{A}\|f\|$ since the dual frame has upper frame bound $A^{-1}$ (see $\S 2.3$ ). Therefore (3.12) gives

$$
\left\|f-\mathcal{P}_{M, N}^{\epsilon} f\right\| \leq\left(1+\sqrt{\frac{B}{A}} \theta\right)\left\|f-\sum_{n \in I_{N}}\left\langle f, \mathcal{S}^{-1} \phi_{n}\right\rangle \phi_{n}\right\|+\epsilon \frac{\theta}{A}\|f\| .
$$

As $N \rightarrow \infty(2.8)$ gives that the first term vanishes. Hence we obtain the result for $f$. For the other results, we use Theorem 3.3 instead.

In summary, provided $M$ is chosen above the stable sampling rate $\Theta^{\epsilon}(N, \theta)$, the approximation $\mathcal{P}_{M, N}^{\epsilon} f$ converges to within roughly $\epsilon$ of $f$, the coefficients converge to within roughly $\epsilon$ of the frame coefficients $\boldsymbol{a}$, and in particular are small in norm for large $N$. Furthermore, the rate of decay of the error is dictated by how well $f$ can be approximated by elements of $\mathrm{H}_{N}$ with bounded coefficients; see (3.12). This latter issue depends completely on the frame $\Phi$ and element $f$ being approximated; see [4] for further discussion.

Note that the behaviour of $\Theta^{\epsilon}(N, \theta)$ as a function of $N$ also depends completely on $\Phi$. We shall consider this issue further in $\S 5$.

\subsection{The need for regularization}

To conclude this section, we now give some brief intuition as to why $\boldsymbol{G}_{M, N}$ is ill-conditioned, even when $M \gg N$. Suppose that $N$ is fixed and $M \rightarrow \infty$. Then, due to the strong convergence of the partial frame operators $\mathcal{S}_{M}=\mathcal{T}_{N} \mathcal{T}_{M}^{*} \rightarrow \mathcal{S}=\mathcal{T} \mathcal{T}^{*}$, one has that $\left(\boldsymbol{G}_{M, N}\right)^{*} \boldsymbol{G}_{M, N} \rightarrow \tilde{\boldsymbol{G}}_{N}$, 
where $\tilde{\boldsymbol{G}}_{N}=\left\{\left\langle\mathcal{S} \phi_{m}, \phi_{n}\right\rangle\right\}_{m, n \in I_{N}}$. This follows directly from the fact that the $(m, n)^{\text {th }}$ entry of $\left(\boldsymbol{G}_{M, N}\right)^{*} \boldsymbol{G}_{M, N}$ is precisely $\left\langle\mathcal{S}_{M} \phi_{m}, \phi_{n}\right\rangle$. Hence

$$
\kappa\left(\boldsymbol{G}_{M, N}\right) \rightarrow \sqrt{\kappa\left(\tilde{\boldsymbol{G}}_{N}\right)}, \quad M \rightarrow \infty .
$$

In particular, if the frame $\Phi$ is tight $(A=B)$, then $\mathcal{S}=A \mathcal{I}$ is a multiple of the identity operator and therefore

$$
\left(\boldsymbol{G}_{M, N}\right)^{*} \boldsymbol{G}_{M, N} \rightarrow A \boldsymbol{G}_{N}, \quad \kappa\left(\boldsymbol{G}_{M, N}\right) \rightarrow \sqrt{\kappa\left(\boldsymbol{G}_{N}\right)}, \quad M \rightarrow \infty,
$$

where $\boldsymbol{G}_{N}=\boldsymbol{G}_{N, N}$. Hence $\boldsymbol{G}_{M, N}$ will inherit the ill-conditioning of $\boldsymbol{G}_{N}$ for large $M$ and $N$. In the non-tight case, recall that operator $\mathcal{S}$ is self-adjoint and positive definite (2.5). Hence it has a unique positive definite square root $\mathcal{S}^{1 / 2}$. This means that

$$
\tilde{\boldsymbol{G}}_{N}=\left\{\left\langle\mathcal{S}^{1 / 2} \phi_{m}, \mathcal{S}^{1 / 2} \phi_{n}\right\rangle\right\}_{m, n \in I_{N}}
$$

is precisely the truncated Gram matrix of the system $\left\{\mathcal{S}^{1 / 2} \phi_{n}\right\}_{n \in I}$. Note that this is a frame, and its frame bounds are readily shown to be $A^{2}$ and $B^{2}$ respectively. Moreover, it is a simple argument via (2.5) to show that $\left\{\mathcal{S}^{1 / 2} \phi_{n}\right\}_{n \in I}$ is a Riesz basis if and only if $\Phi=\left\{\phi_{n}\right\}_{n \in I}$ is a Riesz basis. Therefore, since we assume $\Phi$ is not a Riesz basis throughout, Lemma 2.9 gives that $\tilde{\boldsymbol{G}}_{N}$ is necessarily ill-conditioned for large $N$. Consequently, so are the matrices $\boldsymbol{G}_{M, N}$.

Remark 3.7 It also follows that the projections $\mathcal{P}_{M, N} \rightarrow \mathcal{S}^{-1 / 2} \tilde{\mathcal{P}}_{N}$ strongly as $M \rightarrow \infty$ for fixed $N$, where $\tilde{\mathcal{P}}_{N}$ is the orthogonal projection of $\mathcal{S}^{1 / 2} f$ with respect to the frame $\left\{\mathcal{S}^{1 / 2} \phi_{n}\right\}_{n \in I_{N}}$. In particular, if $\Phi$ is tight then $\mathcal{P}_{M, N} \rightarrow \mathcal{P}_{N}$ as $M \rightarrow \infty$.

This argument gives some insight into the advantage of oversampling. For a tight frame, $\left(\boldsymbol{G}_{M, N}\right)^{*} \boldsymbol{G}_{M, N}$ is an approximate factorization of $\boldsymbol{G}_{N}$ (or $\tilde{\boldsymbol{G}}_{N}$ in the general case). Thus, solving the linear system (1.2) is akin to solving the normal equations of the least-squares problem (3.1). In this sense it is not surprising that oversampling yields $\mathcal{O}(\epsilon)$ accuracy, whereas solving (3.1) yields only $\mathcal{O}(\sqrt{\epsilon})$ accuracy. Indeed, this is reminiscent of the typical squaring of the condition number incurred when forming the normal equations of a least-squares problem $[9, \S 5.3]$.

\section{Approximation from indirect data}

Computing any of the approximations presented so far requires calculation of the inner products $\left\langle f, \phi_{m}\right\rangle$. This is typically inconvenient, since these are often given as integrals. Instead, we want frame approximations that work when $f$ is only specified through a fixed set of indirect data, e.g. pointwise samples. Fortunately, the approached developed in the previous section of oversampling readily extends to this setting. In this section we describe this more general scenario.

\subsection{Preliminaries}

Let $\mathrm{G}$ be a dense subspace of the Hilbert space $\mathrm{H}$ endowed with a norm $\mid\|\cdot\| \cdot \|$. Suppose that $f$, the function we seek to approximate, and $\Phi$, the frame we use, both belong to G. For each $M \in \mathbb{N}$ let $J_{M}$ be an index set of cardinality $\left|J_{M}\right|=M$, and

$$
\ell_{m, M}: \mathrm{G} \rightarrow \mathbb{C}, \quad m \in J_{M},
$$


be a set of linear functionals which are bounded with respect to $\|\cdot\|$, i.e.

$$
\left|\ell_{m, M}(f)\right| \leq c_{m, M}|\|f \mid\|, \quad f \in \mathrm{G} .
$$

The data of $f$ is now given by

$$
\boldsymbol{y}=\left\{\ell_{m, M}(f)\right\}_{m \in J_{M}} .
$$

Write $\mathcal{M}_{M}: \mathrm{G} \rightarrow \mathbb{C}^{M}$ for the mapping $\mathcal{M}_{M} f=\left\{\ell_{m, M}(f)\right\}_{m \in J_{M}}$. As before, the goal is to compute an approximation to $f$ in $\Phi_{N}$ for some $N \leq M$, but now using the data $\mathcal{M}_{M} f$.

In order to make meaningful statements in the general case about the subsequent approximations we define, we shall make two further assumptions. First, we assume that

$$
\left\|\mathcal{M}_{M} g\right\|^{2}=\sum_{m \in J_{M}}\left|\ell_{m, M}(g)\right|^{2}>0, \quad \forall g \in \mathrm{H}_{N}, g \neq 0 .
$$

Second, we require the data to be sufficiently rich to recover $f$ as $M, N \rightarrow \infty$ in a suitable way. In analogy to the frame bounds (2.4), we shall assume that there exist constants $A^{\prime}, B^{\prime}>0$ such that

$$
A^{\prime}\|f\|^{2} \leq \liminf _{M \rightarrow \infty} \sum_{m \in J_{M}}\left|\ell_{m, M}(f)\right|^{2} \leq \limsup _{M \rightarrow \infty} \sum_{m \in J_{M}}\left|\ell_{m, M}(f)\right|^{2} \leq B^{\prime}\|f\|^{2}, \quad \forall f \in \mathrm{G} .
$$

We comment further on the relation between these two assumptions and the constants involved in $\S 4.4$. However, we note in passing that (4.3) implies (4.2) for fixed $N$ and all sufficiently large $M$, since $\mathrm{H}_{N}$ is finite dimensional.

Before going any further, let us mention several examples of this framework:

Example 4.1 Suppose the samples of $f$ are inner products with respect to the frame elements $\phi_{m}$, i.e. $\ell_{m, M}(f)=\left\langle f, \phi_{m}\right\rangle, m=1, \ldots, M$. This is precisely the setting of $\S 3$ and is a special case of the present setup with $\mathrm{G}=\mathrm{H},\|\cdot\|\|=\| \cdot \|$. Note that (4.3) is precisely the frame condition (2.4); in particular, it holds with $A^{\prime}=A$ and $B^{\prime}=B$. Also, (4.2) holds for any $M \geq N$ by Lemma 3.1.

Example 4.2 Let $\Psi=\left\{\psi_{m}\right\}_{m \in J}$ be another frame (or Riesz/orthonormal basis) of $\mathrm{H}$ and consider samples of the form $\ell_{m, M}(f)=\left\langle f, \psi_{m}\right\rangle, m \in J_{M}$, where $\left\{J_{M}\right\}_{M \in \mathbb{N}}$ are nested index sets satisfying $\left|J_{M}\right|=M$ and (2.9). This problem corresponds to sampling according to the frame $\Psi$ and reconstructing in $\Phi$, as in the original framework of generalized sampling [1,2]. In this case, we also have $\mathrm{G}=\mathrm{H}$ and $\|\cdot\|\|=\| \cdot \|$. The condition (4.3) holds with $A^{\prime}, B^{\prime}$ being the frame bounds for $\Psi$.

Example 4.3 Consider a frame for the Hilbert space $\mathrm{H}=\mathrm{L}^{2}(-1,1)$ of square-integrable functions on $(-1,1)$. Suppose that the samples are pointwise evaluations at equispaced points. In this case, we may take $\mathrm{G}=\mathrm{L}^{\infty}(-1,1)$ with its usual norm, and $\ell_{m, M}(f)=\sqrt{2 / M} f(-1+2(m-1) / M)$, $m=1, \ldots, M$. In this case, (4.3) holds with $A^{\prime}=B^{\prime}=1$, since $\sum_{m=1}^{M}\left|\ell_{m, M}(f)\right|^{2}$ is a Riemann sum approximation to $\int_{-1}^{1}|f(x)|^{2} \mathrm{~d} x$.

More generally, if $-1 \leq t_{1, M}<\ldots<t_{M, M} \leq 1$ are (not necessarily equispaced) sample points, then (4.3) holds with $A^{\prime}=B^{\prime}=1$ if

$$
h_{M}=\max _{0, \ldots, M}\left\{t_{m+1, M}-t_{m, M}\right\} \rightarrow 0, \quad M \rightarrow \infty,
$$

where $t_{0, M}=t_{M, M}-2$ and $t_{M+1, M}=t_{1, M}+2$, since in this case choosing the linear functionals as $\ell_{m, M}(f)=\sqrt{\frac{1}{2}\left(t_{m+1, M}-t_{m-1, M}\right)} f\left(t_{m, M}\right)$ gives a convergent approximation $\sum_{m=1}^{M}\left|\ell_{m, M}(f)\right|^{2}$ to $\|f\|^{2}$ as $M \rightarrow \infty$. 
Given $f \in \mathrm{G}$ and data $\mathcal{M}_{M} f$, we construct the approximation $\mathcal{P}_{M, N}^{\epsilon} f$ as follows. First, let

$$
\boldsymbol{G}_{M, N}=\mathcal{M}_{M} \mathcal{T}_{N}=\left\{\ell_{m, M}\left(\phi_{n}\right)\right\}_{m \in J_{M}, n \in I_{N}} \in \mathbb{C}^{M \times N} .
$$

Note (4.2) is equivalent to the condition that $\boldsymbol{G}_{M, N}$ is full rank. However, since $\boldsymbol{G}_{M, N}$ arises from a frame, it will generally be ill-conditioned. Suppose that $\boldsymbol{G}_{M, N}$ has SVD

$$
\boldsymbol{G}_{M, N}=\boldsymbol{U} \boldsymbol{\Sigma} \boldsymbol{V}^{*}
$$

As before, we let $\epsilon>0$ be a parameter, and truncate the singular values of $\boldsymbol{G}_{M, N}$ according to the criterion

$$
\sigma_{n}>\epsilon .
$$

Having done this, we define the coefficient vector $\boldsymbol{x}^{\epsilon}$ as

$$
\boldsymbol{x}^{\epsilon}=\left(\boldsymbol{G}_{M, N}^{\epsilon}\right)^{\dagger} \boldsymbol{y}
$$

We then let

$$
\mathcal{P}_{M, N}^{\epsilon} f=\mathcal{T}_{N} \boldsymbol{x}^{\epsilon}=\mathcal{L}_{M, N}^{\epsilon} \mathcal{M}_{M} f
$$

where $\mathcal{L}_{M, N}^{\epsilon}$ is the mapping

$$
\mathcal{L}_{M, N}^{\epsilon}: \mathbb{C}^{M} \rightarrow \mathrm{H}_{N}, \boldsymbol{y} \mapsto \mathcal{T}_{N}\left(\boldsymbol{G}_{M, N}^{\epsilon}\right)^{\dagger} \boldsymbol{y}
$$

\subsection{The solution as an orthogonal projection}

A key element of our subsequent analysis is the reinterpretation of the operator $\mathcal{P}_{M, N}^{\epsilon}$ as a projection with respect to a suitable inner product. Specifically, we now define the data-dependent sesquilinear form on $\mathrm{G} \times \mathrm{G}$

$$
\langle f, g\rangle_{M}=\left\langle\mathcal{M}_{M} f, \mathcal{M}_{M} g\right\rangle=\sum_{m \in J_{M}} \ell_{m, M}(f) \overline{\ell_{m, M}(g)}, \quad f, g \in \mathrm{G}
$$

with corresponding discrete semi-norm $\|f\|_{M}=\sqrt{\langle f, f\rangle_{M}}=\left\|\mathcal{M}_{M} f\right\|$. Note that (4.2) implies that $\langle\cdot, \cdot\rangle_{M}$ is an inner product on the finite-dimensional subspace $\mathrm{H}_{N}$.

Recall the singular value decomposition (4.4), and let $\boldsymbol{u}_{1}, \ldots, \boldsymbol{u}_{M}, \boldsymbol{v}_{1}, \ldots, \boldsymbol{v}_{N}$ and $\sigma_{1}, \ldots, \sigma_{N}$ be the left and right singular vectors and singular values of $\boldsymbol{G}_{M, N}$ respectively. To the right singular vectors, we associate the functions

$$
\xi_{n}=\mathcal{T}_{N} \boldsymbol{v}_{n} \in \mathrm{H}_{N}, \quad n=1, \ldots, N .
$$

By construction, these functions are orthogonal with respect to the discrete inner product $\langle\cdot, \cdot\rangle_{M}$. Indeed, by orthogonality of the singular vectors, we have

$$
\left\langle\xi_{m}, \xi_{n}\right\rangle_{M}=\left\langle\mathcal{M}_{M} \mathcal{T}_{N} \boldsymbol{v}_{n}, \mathcal{M}_{M} \mathcal{T}_{N} \boldsymbol{v}_{n}\right\rangle=\sigma_{n} \sigma_{m}\left\langle\boldsymbol{u}_{m}, \boldsymbol{u}_{n}\right\rangle=\sigma_{n} \sigma_{m} \delta_{m, n}, \quad m, n \in I_{N}
$$

Furthermore, since $\left\{\boldsymbol{v}_{n}\right\}_{n \in I_{N}}$ is a basis of $\mathbb{C}^{N}$, the functions $\left\{\xi_{n}\right\}_{n \in I_{N}}$ are an orthogonal basis of $\mathrm{H}_{N}$ with respect to the inner product $\langle\cdot, \cdot\rangle_{M}$.

As in $\S 3$, let $\boldsymbol{x}$ be the solution of the unregularized problem, i.e. $\boldsymbol{x}=\left(\boldsymbol{G}_{M, N}\right)^{\dagger} \boldsymbol{y}$. Using the expression for the pseudoinverse in terms of the SVD, we can write both $\boldsymbol{x}$ and the regularized solution $\boldsymbol{x}^{\epsilon}$ in terms of the left and right singular vectors. Specifically,

$$
\boldsymbol{x}=\sum_{n \in I_{N}} \frac{\left\langle\boldsymbol{y}, \boldsymbol{u}_{n}\right\rangle}{\sigma_{n}} \boldsymbol{v}_{n}, \quad \boldsymbol{x}^{\epsilon}=\sum_{\sigma_{n}>\epsilon} \frac{\left\langle\boldsymbol{y}, \boldsymbol{u}_{n}\right\rangle}{\sigma_{n}} \boldsymbol{v}_{n} .
$$


Furthermore, we also have

$$
\left\langle\boldsymbol{y}, \boldsymbol{u}_{n}\right\rangle=\frac{\left\langle\mathcal{M}_{M} f, \boldsymbol{G}_{M, N} \boldsymbol{v}_{n}\right\rangle}{\sigma_{n}}=\frac{\left\langle\mathcal{M}_{M} f, \mathcal{M}_{M} \mathcal{T}_{N} \boldsymbol{v}_{n}\right\rangle}{\sigma_{n}}=\frac{\left\langle f, \xi_{n}\right\rangle_{M}}{\sigma_{n}},
$$

where in the last step we use (4.8). In particular, this gives

$$
\mathcal{P}_{M, N}^{\epsilon} f=\mathcal{T}_{N} \boldsymbol{x}^{\epsilon}=\sum_{\sigma_{n}>\epsilon} \frac{\left\langle\boldsymbol{y}, \boldsymbol{u}_{n}\right\rangle}{\sigma_{n}} \mathcal{T}_{N} \boldsymbol{v}_{n}=\sum_{\sigma_{n}>\epsilon} \frac{\left\langle f, \xi_{n}\right\rangle_{M}}{\sigma_{n}^{2}} \xi_{n}
$$

Similarly, we have

$$
\mathcal{P}_{M, N} f=\mathcal{T}_{N} \boldsymbol{x}=\sum_{n \in I_{N}} \frac{\left\langle f, \xi_{n}\right\rangle_{M}}{\sigma_{n}^{2}} \xi_{n} .
$$

Recalling that $\left\{\xi_{n}\right\}_{n \in I_{N}}$ is an orthogonal basis for $\mathrm{H}_{N}$ with respect to $\langle\cdot, \cdot\rangle_{M}$ we immediately see that $\mathcal{P}_{M, N}$ is the orthogonal projection onto $\mathrm{H}_{N}$ with respect to this inner product. Moreover, $\mathcal{P}_{M, N}^{\epsilon}$ is the orthogonal projectionwith respect to $\langle\cdot, \cdot\rangle_{M}$ onto the subspace

$$
\mathrm{H}_{M, N}^{\epsilon}=\operatorname{span}\left\{\xi_{n}: \sigma_{n}>\epsilon\right\}
$$

\subsection{Analysis}

As in $\S 3$, we define the constants

$$
\kappa_{M, N}^{\epsilon}=\max _{\substack{\boldsymbol{y} \in \operatorname{Ran}\left(\mathcal{M}_{M}\right) \\\|\boldsymbol{y}\|=1}}\left\|\mathcal{L}_{M, N}^{\epsilon} \boldsymbol{y}\right\|, \quad \lambda_{M, N}^{\epsilon}=\epsilon^{-1} \max _{\substack{\boldsymbol{z} \in \mathbb{C}^{N} \\\|\boldsymbol{z}\|=1}}\left\|\mathcal{T}_{N} \boldsymbol{z}-\mathcal{P}_{M, N}^{\epsilon} \mathcal{T}_{N} \boldsymbol{z}\right\|
$$

The only difference between this and (3.5) is in the mapping $\mathcal{L}_{M, N}^{\epsilon}$, which is now given by (4.6), and the restriction of $\boldsymbol{y}$ to the range of $\mathcal{M}_{M}$ (in general, $\operatorname{Ran}\left(\mathcal{M}_{M}\right)$ may not equal $\mathbb{C}^{M}$, although this is true in the case of $\S 3$ since $\mathcal{M}_{M}=\mathcal{T}_{M}^{*}$ ).

The following two results show the approximation error for $\mathcal{P}_{M, N}^{\epsilon} f$ and the coefficients $\boldsymbol{x}^{\epsilon}$. They are generalizations of Theorems 3.2 and 3.3 respectively.

Theorem 4.4. Let $f \in \mathrm{G}$. The truncated $S V D$ approximation $\mathcal{P}_{M, N}^{\epsilon} f$ satisfies

$$
\left\|f-\mathcal{P}_{M, N}^{\epsilon} f\right\| \leq\left\|f-\mathcal{T}_{N} \boldsymbol{z}\right\|+\kappa_{M, N}^{\epsilon}\left\|f-\mathcal{T}_{N} \boldsymbol{z}\right\|_{M}+\epsilon \lambda_{M, N}^{\epsilon}\|\boldsymbol{z}\|, \quad \forall \boldsymbol{z} \in \mathbb{C}^{N} .
$$

Before we present the proof of this result, we first show how it implies Theorem 3.2:

Proof of Theorem 3.2. In this case, $\mathcal{M}_{M}=\mathcal{T}_{M}^{*}$ and therefore $\left\|f-\mathcal{T}_{N} \boldsymbol{z}\right\|_{M}=\left\|\mathcal{T}_{M}^{*}\left(f-\mathcal{T}_{N} \boldsymbol{z}\right)\right\| \leq$ $\sqrt{B}\left\|f-\mathcal{T}_{N} \boldsymbol{z}\right\|$. The result now follows immediately.

The main difference between this result and Theorem 3.2 is that the discrete data norm $\| f-$ $\mathcal{T}_{N} \boldsymbol{z} \|_{M}$ cannot be bounded by $\left\|f-\mathcal{T}_{N} \boldsymbol{z}\right\|$ in general. However, one clearly has

$$
\left\|f-\mathcal{T}_{N} \boldsymbol{z}\right\|_{M} \leq C_{M}\left\|f-\mathcal{T}_{N} \boldsymbol{z}\right\|, \quad C_{M}=\sqrt{\sum_{m \in J_{M}}\left(c_{m, M}\right)^{2}},
$$

where $c_{m, M}$ are the norms of the functionals $\ell_{m, M}$; recall (4.1). In particular, for Example 4.3 it follows that $\left\|f-\mathcal{T}_{N} \boldsymbol{z}\right\|_{M} \leq \sqrt{2}\left\|f-\mathcal{T}_{N} \boldsymbol{z}\right\|_{\mathrm{L}^{\infty}}$. 
Proof of Theorem 4.4. For any $\boldsymbol{z} \in \mathbb{C}^{N}$,

$$
\left\|f-\mathcal{P}_{M, N}^{\epsilon} f\right\| \leq\left\|f-\mathcal{T}_{N} \boldsymbol{z}\right\|+\left\|\mathcal{P}_{M, N}^{\epsilon}\left(f-\mathcal{T}_{N} \boldsymbol{z}\right)\right\|+\left\|\mathcal{T}_{N} \boldsymbol{z}-\mathcal{P}_{M, N}^{\epsilon} \mathcal{T}_{N} \boldsymbol{z}\right\|
$$

Consider the second term. We have

$$
\left\|\mathcal{P}_{M, N}^{\epsilon}\left(f-\mathcal{T}_{N} \boldsymbol{z}\right)\right\|=\left\|\mathcal{L}_{M, N}^{\epsilon} \mathcal{M}_{M}\left(f-\mathcal{T}_{N} \boldsymbol{z}\right)\right\| \leq \kappa_{M, N}^{\epsilon}\left\|\mathcal{M}_{M}\left(f-\mathcal{T}_{N} \boldsymbol{z}\right)\right\|=\kappa_{M, N}^{\epsilon}\left\|f-\mathcal{T}_{N} \boldsymbol{z}\right\|_{M},
$$

which gives the corresponding term in (3.7). Now consider the third term. It follows immediately from the definition of $\lambda_{M, N}^{\epsilon}$ that $\left\|\mathcal{T}_{N} \boldsymbol{z}-\mathcal{P}_{M, N}^{\epsilon} \mathcal{T}_{N} \boldsymbol{z}\right\| \leq \epsilon \lambda_{M, N}^{\epsilon}\|\boldsymbol{z}\|$, as required.

Theorem 4.5. Let $f \in \mathrm{G}$. The coefficients $\boldsymbol{x}^{\epsilon}$ of the truncated $S V D$ projection $\mathcal{P}_{M, N}^{\epsilon}$ satisfy

$$
\left\|\boldsymbol{x}^{\epsilon}\right\| \leq \frac{1}{\epsilon}\left\|f-\mathcal{T}_{N} \boldsymbol{z}\right\|_{M}+\|\boldsymbol{z}\|, \quad \forall \boldsymbol{z} \in \mathbb{C}^{N}
$$

Moreover, if $\boldsymbol{a}=\left\{\left\langle f, \mathcal{S}^{-1} \phi_{n}\right\rangle\right\}_{n \in I}$ are the frame coefficients of $f$ and $\boldsymbol{a}_{M, N}^{\epsilon} \in \ell^{2}(I)$ is the extension of $\boldsymbol{x}^{\epsilon}$ by zero, then

$$
\left\|\boldsymbol{a}-\boldsymbol{a}_{M, N}^{\epsilon}\right\| \leq \sqrt{\sum_{n \in I \backslash I_{N}}\left|a_{n}\right|^{2}}+\frac{1}{\epsilon}\left\|\left(\mathcal{S}-\mathcal{S}_{N}\right) \mathcal{S}^{-1} f\right\|_{M}+\epsilon \frac{\lambda_{M, N}^{\epsilon}}{\sqrt{A}}\|\boldsymbol{a}\| .
$$

Much as above, this result implies Theorem 3.3:

Proof of Theorem 3.3. In this case, we have $\|g\|_{M} \leq \sqrt{B}\|g\|, \forall g \in$ H. Hence (4.14) implies (3.8). For (3.9) we note that

$$
\left\|\left(\mathcal{S}-\mathcal{S}_{N}\right) \mathcal{S}^{-1} f\right\|^{2}=\left\|\sum_{n \in I \backslash I_{N}} a_{n} \phi_{n}\right\|^{2} \leq B \sum_{n \in I \backslash I_{N}}\left|a_{n}\right|^{2}
$$

where for the final step we recall that a frame with upper frame bound $B$ satisfies the upper Riesz basis condition with constant $B$. The result now follows immediately.

For general measurements, (4.15) does not imply convergence of the coefficients $\boldsymbol{a}_{M, N}^{\epsilon}$ to the frame coefficients $\boldsymbol{a}$ since the term $\left\|\left(\mathcal{S}-\mathcal{S}_{N}\right) \mathcal{S}^{-1} f\right\|_{M}$ cannot be bounded by $\left\|\left(\mathcal{S}-\mathcal{S}_{N}\right) \mathcal{S}^{-1} f\right\|$ in general. There is also no guarantee that $\left\|\left(\mathcal{S}-\mathcal{S}_{N}\right) \mathcal{S}^{-1} f\right\| \rightarrow 0$ as $N \rightarrow \infty$. This does hold, however, when the data arises from sampling with another frame $\left\{\psi_{n}\right\}_{n \in I}$ (Example 4.2). In this case, $\mathcal{M}_{M}$ is the truncated analysis operator of this frame, the constants $A^{\prime}, B^{\prime}$ in (4.3) are the frame bounds, and therefore $\|g\|_{M} \leq B^{\prime}\|g\|, \forall g \in \mathrm{H}$.

Proof of Theorem 4.5. For the first part, we use (4.10) to write

$$
\boldsymbol{x}^{\epsilon}=\sum_{\sigma_{n}>\epsilon} \frac{\left\langle f, \xi_{n}\right\rangle_{M}}{\sigma_{n}^{2}} \boldsymbol{v}_{n}=\sum_{\sigma_{n}>\epsilon} \frac{\left\langle f-\mathcal{T}_{N} \boldsymbol{z}, \xi_{n}\right\rangle_{M}}{\sigma_{n}^{2}} \boldsymbol{v}_{n}+\sum_{\sigma_{n}>\epsilon} \frac{\left\langle\mathcal{T}_{N} \boldsymbol{z}, \xi_{n}\right\rangle_{M}}{\sigma_{n}^{2}} \boldsymbol{v}_{n}
$$

Consider the first term on the right-hand side. Since the $\boldsymbol{v}_{n}$ are orthonormal, we have

$$
\left\|\sum_{\sigma_{n}>\epsilon} \frac{\left\langle f-\mathcal{T}_{N} \boldsymbol{z}, \xi_{m}\right\rangle_{M}}{\sigma_{n}^{2}} \boldsymbol{v}_{n}\right\|^{2}=\sum_{\sigma_{n}>\epsilon} \frac{\left|\left\langle f-\mathcal{T}_{N} \boldsymbol{z}, \xi_{m}\right\rangle_{M}\right|^{2}}{\sigma_{m}^{4}} \leq \frac{1}{\epsilon^{2}} \sum_{\sigma_{n}>\epsilon} \frac{\left|\left\langle f-\mathcal{T}_{N} \boldsymbol{z}, \xi_{m}\right\rangle_{M}\right|^{2}}{\sigma_{m}^{2}} .
$$


It follows from (4.9) and (4.11) that

$$
\sum_{\sigma_{n}>\epsilon} \frac{\left|\left\langle g, \xi_{m}\right\rangle_{M}\right|^{2}}{\sigma_{m}^{2}}=\left\|\mathcal{P}_{M, N}^{\epsilon} g\right\|_{M}^{2}, \quad g \in \mathrm{G} .
$$

Hence

$$
\left\|\sum_{\sigma_{n}>\epsilon} \frac{\left\langle f-\mathcal{T}_{N} \boldsymbol{z}, \xi_{m}\right\rangle_{M}}{\sigma_{n}^{2}} \boldsymbol{v}_{n}\right\|^{2} \leq \frac{1}{\epsilon^{2}}\left\|\mathcal{P}_{M, N}^{\epsilon}\left(f-\mathcal{T}_{N} \boldsymbol{z}\right)\right\|_{M} \leq \frac{1}{\epsilon^{2}}\left\|f-\mathcal{T}_{N} \boldsymbol{z}\right\|_{M}^{2},
$$

where in the second step we use the fact that $\mathcal{P}_{M, N}^{\epsilon}$ is the orthogonal projection with respect to $\langle\cdot, \cdot\rangle_{M}$. This gives the first term of first term of (4.14). Next, consider the second term of the right-hand side of (4.16). Since

$$
\left\langle\mathcal{T}_{N} \boldsymbol{z}, \xi_{m}\right\rangle_{M}=\left\langle\mathcal{T}_{N} \boldsymbol{z}, \mathcal{T}_{N} \boldsymbol{v}_{n}\right\rangle_{M}=\sigma_{n}^{2}\left\langle\boldsymbol{z}, \boldsymbol{v}_{n}\right\rangle,
$$

it follows that

$$
\left\|\sum_{\sigma_{n}>\epsilon} \frac{\left\langle\mathcal{T}_{N} \boldsymbol{z}, \xi_{m}\right\rangle_{M}}{\sigma_{n}^{2}} \boldsymbol{v}_{n}\right\|^{2}=\sum_{\sigma_{n}>\epsilon}\left|\left\langle\boldsymbol{z}, \boldsymbol{v}_{n}\right\rangle\right|^{2} \leq\|\boldsymbol{z}\|,
$$

This gives the second term of (4.14).

For (4.15) we first note that

$$
\left\|\boldsymbol{a}-\boldsymbol{a}_{M, N}^{\epsilon}\right\| \leq \sqrt{\sum_{n \in I \backslash I_{N}}\left|a_{n}\right|^{2}}+\left\|\boldsymbol{a}_{N}-\boldsymbol{x}^{\epsilon}\right\|,
$$

where $\boldsymbol{a}_{N}=\left\{a_{n}\right\}_{n \in I_{N}}$. Therefore it suffices to consider $\left\|\boldsymbol{a}_{N}-\boldsymbol{x}^{\epsilon}\right\|$. Observe that

$$
\boldsymbol{a}_{N}=\sum_{n \in I_{N}}\left\langle\boldsymbol{a}_{N}, \boldsymbol{v}_{n}\right\rangle \boldsymbol{v}_{n}=\sum_{n \in I_{N}}\left\langle\mathcal{S}^{-1} f, \xi_{n}\right\rangle \boldsymbol{v}_{n}
$$

Now $\left\langle f, \xi_{n}\right\rangle_{M}=\left\langle\mathcal{S S}^{-1} f, \xi_{n}\right\rangle_{M}$ and therefore

$$
\left\langle f, \xi_{n}\right\rangle_{M}=\left\langle\mathcal{S}_{N} \mathcal{S}^{-1} f, \xi_{n}\right\rangle_{M}+\left\langle\left(\mathcal{S}-\mathcal{S}_{N}\right) \mathcal{S}^{-1} f, \xi_{n}\right\rangle_{M}=\sigma_{n}^{2}\left\langle\mathcal{T}_{N}^{*} \mathcal{S}^{-1} f, \boldsymbol{v}_{n}\right\rangle+\left\langle\left(\mathcal{S}-\mathcal{S}_{N}\right) \mathcal{S}^{-1} f, \xi_{n}\right\rangle_{M} .
$$

Notice that $\mathcal{S}_{N} \mathcal{S}^{-1} f \in \mathrm{G}$ and $\left(\mathcal{S}-\mathcal{S}_{N}\right) \mathcal{S}^{-1} f=f-\mathcal{S}_{N} \mathcal{S}^{-1} f \in \mathrm{G}$. Therefore all the terms above are well defined. Hence, by (4.16),

$$
\boldsymbol{x}^{\epsilon}=\sum_{\sigma_{n}>\epsilon}\left\langle\mathcal{S}^{-1} f, \xi_{n}\right\rangle \boldsymbol{v}_{n}+\sum_{\sigma_{n}>\epsilon} \frac{\left\langle\left(\mathcal{S}-\mathcal{S}_{M}\right) \mathcal{S}^{-1} f, \xi_{m}\right\rangle_{N}}{\sigma_{n}^{2}} \boldsymbol{v}_{n},
$$

which gives

$$
\left\|\boldsymbol{a}_{N}-\boldsymbol{x}^{\epsilon}\right\| \leq\left\|\sum_{\sigma_{n} \leq \epsilon}\left\langle\mathcal{S}^{-1} f, \xi_{n}\right\rangle \boldsymbol{v}_{n}\right\|+\left\|\sum_{\sigma_{n}>\epsilon} \frac{\left\langle\left(\mathcal{S}-\mathcal{S}_{M}\right) \mathcal{S}^{-1} f, \xi_{n}\right\rangle_{M}}{\sigma_{n}^{2}} \boldsymbol{v}_{n}\right\| .
$$

Consider the first term. Let $\boldsymbol{z} \in \mathbb{C}^{N}$ be given by $\boldsymbol{z}=\sum_{\sigma_{n} \leq \epsilon}\left\langle\mathcal{S}^{-1} f, \xi_{n}\right\rangle \boldsymbol{v}_{n}$, so that the first term is merely $\|\boldsymbol{z}\|$. By the definition of $\lambda_{M, N}^{\epsilon}$, we have $\epsilon \lambda_{M, N}^{\epsilon}\|\boldsymbol{z}\| \geq\left\|\mathcal{T}_{N} \boldsymbol{z}-\mathcal{P}_{M, N}^{\epsilon} \mathcal{T}_{N} \boldsymbol{z}\right\|$. Now, since $\mathcal{T}_{N} \boldsymbol{z} \perp \mathrm{H}_{M, N}^{\epsilon}$, we have that $\mathcal{P}_{M, N}^{\epsilon} \mathcal{T}_{N} \boldsymbol{z}=0$. Hence

$$
\epsilon \lambda_{M, N}^{\epsilon}\|\boldsymbol{z}\| \geq\left\|\mathcal{T}_{N} \boldsymbol{z}\right\|=\sup _{\substack{g \in \mathrm{H} \\ g \neq 0}} \frac{\left|\left\langle\mathcal{T}_{N} \boldsymbol{z}, g\right\rangle\right|}{\|g\|} .
$$


Set $g=\mathcal{S}^{-1} f$. Then $\left\langle\mathcal{T}_{N} \boldsymbol{z}, g\right\rangle=\sum_{\sigma_{n} \leq \epsilon}\left|\left\langle\mathcal{S}^{-1} f, \xi_{m}\right\rangle\right|^{2}=\|\boldsymbol{z}\|^{2}$ and therefore we obtain $\epsilon \lambda_{M, N}^{\epsilon}\|\boldsymbol{z}\| \geq$ $\|\boldsymbol{z}\|^{2} /\left\|\mathcal{S}^{-1} f\right\|$. It follows that

$$
\|\boldsymbol{z}\|=\left\|\sum_{\sigma_{n} \leq \epsilon}\left\langle\mathcal{S}^{-1} f, \xi_{m}\right\rangle \boldsymbol{v}_{n}\right\| \leq \epsilon \lambda_{M, N}^{\epsilon}\left\|\mathcal{S}^{-1} f\right\| \leq \epsilon \lambda_{M, N}^{\epsilon} / \sqrt{A}\|\boldsymbol{a}\| .
$$

Now consider the second term of (4.18). We have

$$
\begin{aligned}
\left\|\sum_{\sigma_{n}>\epsilon} \frac{\left\langle\left(\mathcal{S}-\mathcal{S}_{M}\right) \mathcal{S}^{-1} f, \xi_{n}\right\rangle_{M}}{\sigma_{n}^{2}} \boldsymbol{v}_{n}\right\|^{2}=\sum_{\sigma_{n}>\epsilon} \frac{\left|\left\langle\left(\mathcal{S}-\mathcal{S}_{N}\right) \mathcal{S}^{-1} f, \xi_{n}\right\rangle_{M}\right|^{2}}{\sigma_{n}^{4}} & \leq \frac{1}{\epsilon^{2}}\left\|\mathcal{P}_{M, N}^{\epsilon}\left(\mathcal{S}-\mathcal{S}_{N}\right) \mathcal{S}^{-1} f\right\|_{M}^{2} \\
& \leq \frac{1}{\epsilon^{2}}\left\|\left(\mathcal{S}-\mathcal{S}_{N}\right) \mathcal{S}^{-1} f\right\|_{M}^{2}
\end{aligned}
$$

Combining this with (4.19) now gives the result.

\subsection{Behaviour of the constants and the stable sampling rate}

We now consider the behaviour of the constants $\kappa_{M, N}^{\epsilon}$ and $\lambda_{M, N}^{\epsilon}$. To do so, we define the constant $A_{M, N}^{\prime}$ as follows:

$$
A_{M, N}^{\prime}=\inf _{\substack{g \in \mathrm{H}_{N} \\\|g\|=1}}\|g\|_{M}^{2}
$$

The assumption (4.2) implies that $A_{M, N}^{\prime}>0$. Moreover, due to (4.3) and the fact that $\mathrm{H}_{M}$ is finite-dimensional, we have that $\liminf _{M \rightarrow \infty} A_{M, N}^{\prime} \geq A^{\prime}$ for any fixed $N$.

Proposition 4.6. The constants $\kappa_{M, N}^{\epsilon}$ and $\lambda_{M, N}^{\epsilon}$ defined in (4.12) satisfy

$$
\kappa_{M, N}^{\epsilon} \leq \frac{\sqrt{B}}{\epsilon}, \quad \lambda_{M, N}^{\epsilon} \leq \frac{\sqrt{B}}{\epsilon}, \quad \forall M, N \in \mathbb{N}, M \geq N
$$

Moreover,

$$
\kappa_{M, N}^{\epsilon} \leq \frac{1}{\sqrt{A_{M, N}^{\prime}}}, \quad \lambda_{M, N}^{\epsilon} \leq \frac{1}{\sqrt{A_{M, N}^{\prime}}} .
$$

In particular, for fixed $N$, $\lim \sup _{M \rightarrow \infty} \kappa_{M, N}^{\epsilon} \leq \frac{1}{\sqrt{A^{\prime}}}$ and $\lim \sup _{M \rightarrow \infty} \lambda_{M, N}^{\epsilon} \leq \frac{1}{\sqrt{A^{\prime}}}$.

Proof. Let $\boldsymbol{y} \in \operatorname{Ran}\left(\mathcal{M}_{M}\right)$ be given and write $\boldsymbol{y}=\mathcal{M}_{M} f$ for some $f \in \mathrm{G}$. Then, by (4.11),

$$
\left\|\mathcal{L}_{M, N}^{\epsilon} \boldsymbol{y}\right\|=\left\|\mathcal{P}_{M, N}^{\epsilon} f\right\|=\left\|\mathcal{T}_{N} \sum_{\sigma_{n}>\epsilon} \frac{\left\langle\boldsymbol{y}, \boldsymbol{u}_{n}\right\rangle}{\sigma_{n}} \boldsymbol{v}_{n}\right\|
$$

Recall that $\left\|\mathcal{T}_{N} \boldsymbol{x}\right\| \leq \sqrt{B}\|\boldsymbol{x}\|$ since $\Phi_{N}$ is a Riesz basis for $\mathrm{H}_{N}$. Hence

$$
\left\|\mathcal{P}_{M, N}^{\epsilon} f\right\|^{2} \leq B \sum_{\sigma_{n}>\epsilon} \frac{\left|\left\langle\boldsymbol{y}, \boldsymbol{u}_{n}\right\rangle\right|^{2}}{\sigma_{n}^{2}} \leq \frac{B}{\epsilon^{2}}\|\boldsymbol{y}\|^{2},
$$

which gives (4.21) for $\kappa_{M, N}^{\epsilon}$. For (4.22), we let $\boldsymbol{y} \in \operatorname{Ran}\left(\mathcal{M}_{M}\right)$ and write $\boldsymbol{y}=\mathcal{M}_{M} f$ for some $f \in \mathrm{G}$ once more. Since $\mathcal{P}_{M, N}^{\epsilon} f$ is an orthogonal projection with respect to $\langle\cdot, \cdot\rangle_{M}$ we have

$$
\left\|\mathcal{P}_{M, N}^{\epsilon} f\right\|_{M}^{2}=\left\langle f, \mathcal{P}_{M, N}^{\epsilon} f\right\rangle_{M}
$$


In particular, $\left\|\mathcal{P}_{M, N}^{\epsilon} f\right\|_{M} \leq\|f\|_{M}$. Hence

$$
\left\|\mathcal{P}_{M, N}^{\epsilon} f\right\| \leq \frac{1}{\sqrt{A_{M, N}^{\prime}}}\left\|\mathcal{P}_{M, N}^{\epsilon} f\right\|_{M} \leq \frac{1}{\sqrt{A_{M, N}^{\prime}}}\|f\|_{M}=\frac{1}{\sqrt{A_{M, N}^{\prime}}}\|\boldsymbol{y}\| .
$$

This gives (4.22).

We now consider $\lambda_{M, N}^{\epsilon}$. Let $\boldsymbol{z} \in \mathbb{C}^{M}$ be arbitrary. Using (4.11) and (4.17) we have

$$
\mathcal{T}_{N} \boldsymbol{z}-\mathcal{P}_{M, N}^{\epsilon} \mathcal{T}_{N} \boldsymbol{z}=\mathcal{T}_{N} \sum_{\sigma_{n} \leq \epsilon}\left\langle\boldsymbol{z}, \boldsymbol{v}_{n}\right\rangle \boldsymbol{v}_{n}
$$

Arguing as above, this implies that $\left\|\mathcal{T}_{N} \boldsymbol{z}-\mathcal{P}_{M, N}^{\epsilon} \mathcal{T}_{N} \boldsymbol{z}\right\|^{2} \leq B\|\boldsymbol{z}\|^{2}$, which gives (4.21). For (4.22), we again let $\boldsymbol{z} \in \mathbb{C}^{M}$ be arbitrary. Then

$$
\left\|\mathcal{T}_{N} \boldsymbol{z}-\mathcal{P}_{M, N}^{\epsilon} \mathcal{T}_{N} \boldsymbol{z}\right\|_{M}^{2}=\sum_{\sigma_{n} \leq \epsilon} \sigma_{n}^{2}\left|\left\langle\boldsymbol{z}, \boldsymbol{v}_{n}\right\rangle\right|^{2} \leq \epsilon^{2}\|\boldsymbol{z}\|^{2}
$$

Moreover, since $\mathcal{T}_{N} \boldsymbol{z}-\mathcal{P}_{M, N}^{\epsilon} \mathcal{T}_{N} \boldsymbol{z} \in \mathrm{H}_{N}$ we obtain

$$
\left\|\mathcal{T}_{N} \boldsymbol{z}-\mathcal{P}_{M, N}^{\epsilon} \mathcal{T}_{N} \boldsymbol{z}\right\|^{2} \leq \frac{1}{A_{M, N}^{\prime}}\left\|\mathcal{T}_{N} \boldsymbol{z}-\mathcal{P}_{M, N}^{\epsilon}\right\|_{M}^{2} \leq \frac{\epsilon^{2}}{A_{M, N}^{\prime}}\|\boldsymbol{z}\|^{2}
$$

as required.

Proof of Proposition 3.4. The second part of this lemma follows immediately from Proposition 4.6, recalling that $A^{\prime}=A$ and $B^{\prime}=B$ in this case. For the first part, we argue in a slightly different way.

Consider $\kappa_{M, N}^{\epsilon}$ first. Let $\boldsymbol{y} \in \mathbb{C}^{M}$ be given and write $\boldsymbol{y}=\mathcal{T}_{M}^{*} f$ for some $f \in \mathrm{H}$ so that $\left\|\mathcal{L}_{M, N}^{\epsilon} \boldsymbol{y}\right\|=\left\|\mathcal{P}_{M, N}^{\epsilon} f\right\|$. By (4.10) we have

$$
\left\|\mathcal{P}_{M, N}^{\epsilon} f\right\|^{2}=\sum_{\sigma_{m}, \sigma_{n}>\epsilon} \frac{\left\langle\boldsymbol{y}, \boldsymbol{u}_{m}\right\rangle \overline{\left\langle\boldsymbol{y}, \boldsymbol{u}_{n}\right\rangle}}{\sigma_{m} \sigma_{n}}\left\langle\xi_{m}, \xi_{n}\right\rangle .
$$

Now $\left\langle\xi_{m}, \xi_{n}\right\rangle=\left\langle\mathcal{T}_{N}^{*} \mathcal{T}_{N} \boldsymbol{v}_{m}, \boldsymbol{v}_{n}\right\rangle=\sigma_{m}\left\langle\tilde{\boldsymbol{u}}_{m}, \boldsymbol{v}_{n}\right\rangle$, where $\tilde{\boldsymbol{u}}_{m} \in \mathbb{C}^{N}$ is the vector with entries $\left(\tilde{\boldsymbol{u}}_{m}\right)_{k}=$ $\left(\boldsymbol{u}_{m}\right)_{k}$ for $k \in I_{N}$. Hence,

$$
\left\|\mathcal{P}_{M, N}^{\epsilon} f\right\|^{2}=\left\langle\sum_{\sigma_{n}>\epsilon}\left\langle\boldsymbol{y}, \boldsymbol{u}_{m}\right\rangle \tilde{\boldsymbol{u}}_{m}, \sum_{\sigma_{n}>\epsilon} \frac{\left\langle\boldsymbol{y}, \boldsymbol{u}_{n}\right\rangle}{\sigma_{n}} \boldsymbol{v}_{n}\right\rangle \leq\left\|\sum_{\sigma_{n}>\epsilon}\left\langle\boldsymbol{y}, \boldsymbol{u}_{m}\right\rangle \tilde{\boldsymbol{u}}_{m}\right\|\left\|\sum_{\sigma_{n}>\epsilon} \frac{\left\langle\boldsymbol{y}, \boldsymbol{u}_{n}\right\rangle}{\sigma_{n}} \boldsymbol{v}_{n}\right\| .
$$

By orthogonality, the second term satisfies

$$
\left\|\sum_{\sigma_{n}>\epsilon} \frac{\left\langle\boldsymbol{y}, \boldsymbol{u}_{n}\right\rangle}{\sigma_{n}} \boldsymbol{v}_{n}\right\| \leq \sqrt{\sum_{\sigma_{n}>\epsilon} \frac{\left|\left\langle\boldsymbol{y}, \boldsymbol{u}_{n}\right\rangle\right|^{2}}{\sigma_{n}^{2}}} \leq \frac{\|\boldsymbol{y}\|}{\epsilon} .
$$

Consider the first term. Let $\mathcal{Q}_{N}: \mathbb{C}^{M} \rightarrow \mathbb{C}^{M}$ be the projection defined by $\left(\mathcal{Q}_{N} \boldsymbol{x}\right)_{m}=x_{m}, m \in I_{N}$ and $\left(\mathcal{Q}_{N} \boldsymbol{x}\right)_{m}=0, m \in I_{M} \backslash I_{N}$. Then

$$
\left\|\sum_{\sigma_{n}>\epsilon}\left\langle\boldsymbol{y}, \boldsymbol{u}_{m}\right\rangle \tilde{\boldsymbol{u}}_{m}\right\|=\left\|\mathcal{Q}_{N}\left(\sum_{\sigma_{n}>\epsilon}\left\langle\boldsymbol{y}, \boldsymbol{u}_{m}\right\rangle \boldsymbol{u}_{m}\right)\right\| \leq\left\|\sum_{\sigma_{n}>\epsilon}\left\langle\boldsymbol{y}, \boldsymbol{u}_{m}\right\rangle \boldsymbol{u}_{m}\right\| \leq\|\boldsymbol{y}\| .
$$


Therefore, we deduce that

$$
\left\|\mathcal{P}_{M, N}^{\epsilon} f\right\|^{2} \leq\|\boldsymbol{y}\| / \epsilon
$$

and the result for $\kappa_{M, N}^{\epsilon}$ now follows from its definition.

Now consider $\lambda_{M, N}^{\epsilon}$. Let $\boldsymbol{z} \in \mathbb{C}^{N}$ be arbitrary and recall that

$$
\mathcal{T}_{N} \boldsymbol{z}-\mathcal{P}_{M, N}^{\epsilon} \mathcal{T}_{N} \boldsymbol{z}=\sum_{\sigma_{n} \leq \epsilon}\left\langle\boldsymbol{z}, \boldsymbol{v}_{n}\right\rangle \xi_{n}
$$

Hence

$$
\left\|\mathcal{T}_{N} \boldsymbol{z}-\mathcal{P}_{M, N}^{\epsilon} \mathcal{T}_{N} \boldsymbol{z}\right\|^{2}=\sum_{\sigma_{m}, \sigma_{n} \leq \epsilon}\left\langle\boldsymbol{z}, \boldsymbol{v}_{n}\right\rangle \overline{\left\langle\boldsymbol{z}, \boldsymbol{v}_{n}\right\rangle}\left\langle\xi_{m}, \xi_{n}\right\rangle .
$$

As above, we note that $\left\langle\xi_{m}, \xi_{n}\right\rangle=\sigma_{m}\left\langle\tilde{\boldsymbol{u}}_{m}, \boldsymbol{v}_{n}\right\rangle$, and therefore

$$
\left\|\mathcal{T}_{N} \boldsymbol{z}-\mathcal{P}_{M, N}^{\epsilon} \mathcal{T}_{N} \boldsymbol{z}\right\|^{2}=\left\langle\sum_{\sigma_{n} \leq \epsilon} \sigma_{m}\left\langle\boldsymbol{z}, \boldsymbol{v}_{m}\right\rangle \tilde{\boldsymbol{u}}_{m}, \sum_{\sigma_{n} \leq \epsilon}\left\langle\boldsymbol{z}, \boldsymbol{v}_{n}\right\rangle \boldsymbol{v}_{n}\right\rangle \leq \epsilon\|\boldsymbol{z}\|^{2} .
$$

Since $\boldsymbol{z}$ was arbitrary, we now obtain the result for $\lambda_{M, N}^{\epsilon}$.

Remark 4.7 Proposition 4.6 gives a more pessimistic bound (4.21) than the corresponding bound (3.10) for the oversampling setting of $\S 3$. Specifically, the bound is proportional to $1 / \epsilon$ as opposed to $1 / \sqrt{\epsilon}$. As is made clear by the proofs, this discrepancy is due to the fact that in the latter case the measurements are just inner products with respect to the same frame.

As in $\S 3$, we may now define the stable sampling rate:

$$
\Theta^{\epsilon}(N, \theta)=\min \left\{M \in \mathbb{N}: M \geq N, \kappa_{M, N}^{\epsilon} \leq \frac{\theta}{\sqrt{A^{\prime}}}, \lambda_{M, N}^{\epsilon} \leq \frac{\theta}{\sqrt{A^{\prime}}}\right\}, \quad 1<\theta<\infty, N \in \mathbb{N} .
$$

Note that sampling at this rate, i.e. setting $M \geq \Theta^{\epsilon}(N, \theta)$, guarantees an error bound of the form

$$
\left\|f-\mathcal{P}_{M, N}^{\epsilon} f\right\| \leq\left\|f-\mathcal{T}_{N} \boldsymbol{z}\right\|+\frac{\theta}{\sqrt{A^{\prime}}}\left\|f-\mathcal{T}_{N} \boldsymbol{z}\right\|_{M}+\epsilon \frac{\theta}{\sqrt{A^{\prime}}}\|\boldsymbol{z}\|, \quad \forall \boldsymbol{z} \in \mathbb{C}^{N},
$$

and therefore decay of the error down to roughly $\epsilon$ as $N \rightarrow \infty$, provided the term $\left\|f-\mathcal{T}_{N} \boldsymbol{z}\right\|_{M} \rightarrow 0$ (see the discussion after Theorem 4.4).

\subsection{Computing the stable sampling rate}

As noted, in order to achieve a well-conditioned and accurate approximation, one needs to determine $\Theta^{\epsilon}(N, \theta)$. One approach to do this is to analyze $\kappa_{M, N}^{\epsilon}$ and $\lambda_{M, N}^{\epsilon}$ for each given frame. We shall see an example of this in $\S 5$. However, this can be difficult for a general frame, and usually only reveals the asymptotic growth rate of $\Theta^{\epsilon}(N, \theta)$ with $N$ and not the precise constant.

Another approach is to compute $\Theta^{\epsilon}(N, \theta)$. For this, we note the following:

Lemma 4.8. The constant $\kappa_{M, N}^{\epsilon}$ satisfies

$$
\kappa_{M, N}^{\epsilon} \leq \sqrt{\lambda_{\max }\left(\left(\boldsymbol{B}_{M, N}^{\epsilon}\right)^{*} \boldsymbol{G}_{N} \boldsymbol{B}_{M, N}^{\epsilon}\right)},
$$


where $\boldsymbol{B}_{M, N}^{\epsilon}=\left(\boldsymbol{G}_{M, N}^{\epsilon}\right)^{\dagger}$. If $\operatorname{Ran}\left(\mathcal{M}_{M}\right)=\mathbb{C}^{M}$ this holds with equality. The constant $\lambda_{M, N}^{\epsilon}$ satisfies

$$
\lambda_{M, N}^{\epsilon}=\epsilon^{-1} \sqrt{\lambda_{\max }\left(\left(\boldsymbol{C}_{M, N}^{\epsilon}\right)^{*} \boldsymbol{G}_{N} \boldsymbol{C}_{M, N}^{\epsilon}\right)}
$$

where $\boldsymbol{C}_{M, N}^{\epsilon}=\boldsymbol{V}\left(\boldsymbol{I}-\boldsymbol{I}^{\epsilon}\right) \boldsymbol{V}^{*}$ and $\boldsymbol{I}_{\epsilon}$ is the diagonal matrix with $n^{\text {th }}$ entry 1 if $\sigma_{n} \geq \epsilon$ and zero otherwise.

Proof. Let $\boldsymbol{y} \in \operatorname{Ran}\left(\mathcal{M}_{M}\right)$ with $\|\boldsymbol{y}\|=1$. Then

$$
\left\|\mathcal{L}_{M, N}^{\epsilon} \boldsymbol{y}\right\|^{2}=\left\|\mathcal{T}_{N} \boldsymbol{B}_{M, N}^{\epsilon} \boldsymbol{y}\right\|^{2}=\boldsymbol{y}^{*}\left(\mathcal{B}_{M, N}^{\epsilon}\right)^{*} \boldsymbol{G}_{N} \boldsymbol{B}_{M, N}^{\epsilon} \boldsymbol{y} \leq \lambda_{\max }\left(\left(\boldsymbol{B}_{M, N}^{\epsilon}\right)^{*} \boldsymbol{G}_{N} \boldsymbol{B}_{M, N}^{\epsilon}\right),
$$

since $\boldsymbol{G}_{N}=\mathcal{T}_{N}^{*} \mathcal{T}_{N}$. This gives the first result.

Let $\boldsymbol{z} \in \mathbb{C}^{N},\|\boldsymbol{z}\|=1$. Then

$$
\begin{aligned}
\left\|\mathcal{T}_{N} \boldsymbol{z}-\mathcal{P}_{M, N}^{\epsilon} \mathcal{T}_{N} \boldsymbol{z}\right\|^{2}=\left\|\mathcal{T}_{N}\left(\boldsymbol{I}-\left(\boldsymbol{G}_{M, N}^{\epsilon}\right)^{\dagger} \boldsymbol{G}_{M, N}\right) \boldsymbol{z}\right\|^{2} & =\left\|\mathcal{T}_{N}\left(\boldsymbol{I}-\boldsymbol{V} \boldsymbol{I}^{\epsilon} \boldsymbol{V}^{*}\right) \boldsymbol{z}\right\|^{2} \\
& =\left\|\mathcal{T}_{N} \boldsymbol{C}_{M, N}^{\epsilon} \boldsymbol{z}\right\|^{2}=\boldsymbol{z}^{*}\left(\boldsymbol{C}_{M, N}^{\epsilon}\right)^{*} \boldsymbol{G}_{N} \boldsymbol{C}_{M, N}^{\epsilon} \boldsymbol{z}
\end{aligned}
$$

Maximizing over $\boldsymbol{z}$ now gives the result.

Hence, both $\kappa_{M, N}^{\epsilon}$ and $\lambda_{M, N}^{\epsilon}$ can be computed, provided $\boldsymbol{G}_{N}$ is known.

Remark 4.9 In practice, it may be difficult to compute $\boldsymbol{G}_{N}$, since its entries are inner products which may for instance be integrals. This may be overcome by a further approximation, e.g. a quadrature. Specifically, let $K \geq 1$ and $\jmath_{k, K}$ be a family of linear functionals such that

$$
\lim _{K \rightarrow \infty} \sum_{k=1}^{K} \jmath_{k, K}(f) \overline{\jmath_{k, K}(g)}=\langle f, g\rangle, \quad \forall f, g \in \mathrm{G} .
$$

Let $\boldsymbol{H}_{K, N}=\left\{\jmath_{k, K}\left(\phi_{n}\right)\right\}_{k=1, n \in I_{N}}^{K} \in \mathbb{C}^{K \times N}$. Then $\left(\boldsymbol{H}_{K, N}\right)^{*} \boldsymbol{H}_{K, N} \approx \boldsymbol{G}_{N}$ for large $K$. Hence, by the previous lemma (assuming $\operatorname{Ran}\left(\mathcal{M}_{M}\right)=\mathbb{C}^{M}$ for ease of presentation), we have

$$
\kappa_{M, N}^{\epsilon} \approx\left\|\boldsymbol{H}_{K, N} \boldsymbol{B}_{M, N}^{\epsilon}\right\|_{2}=\left\|\boldsymbol{H}_{K, N} \boldsymbol{V}\left(\boldsymbol{\Sigma}^{\epsilon}\right)^{\dagger}\right\|_{2}, \quad \lambda_{M, N}^{\epsilon} \approx\left\|\boldsymbol{H}_{K, N} \boldsymbol{V}\left(\boldsymbol{I}-\boldsymbol{I}^{\epsilon}\right)\right\|_{2},
$$

for sufficiently large $K$. If, for instance, the functionals $\jmath_{k, K}$ correspond to pointwise evaluations as part of a quadrature, this gives a means of numerically approximating $\kappa_{M, N}^{\epsilon}$ and $\lambda_{M, N}^{\epsilon}$.

\section{$5 \quad \mathrm{ONB}+1$ and $\mathrm{ONB}+K$ frames}

We conclude this paper with several examples to illustrate the stable sampling rate. Let $\left\{\varphi_{n}\right\}_{n \in \mathbb{N}}$ be an orthonormal basis of $\mathrm{H}$ and $\psi \in \mathrm{H},\|\psi\|=1$, be such that $\left\langle\psi, \varphi_{n}\right\rangle \neq 0$ for infinitely-many $n \in \mathbb{N}$. Then the indexed family

$$
\Phi=\left\{\phi_{0}, \phi_{1}, \ldots\right\}=\left\{\psi, \varphi_{1}, \varphi_{2}, \ldots\right\},
$$

is a linearly-independent frame for $\Phi$ with frame bounds

$$
A=1, \quad B=2 .
$$


We refer to this frame as the $O N B+1$ frame. Note that it was previously used in [4] to show that the Gram matrix of a frame can be arbitrarily badly conditioned. It is motivated by the idea of 'enriching' an orthonormal basis to better capture a certain feature of a function under approximation (e.g. a singularity or oscillation).

Throughout this section, we let $\mathcal{Q}_{N}$ denote the projection onto $\operatorname{span}\left\{\varphi_{1}, \ldots, \varphi_{N}\right\}$, i.e.

$$
\mathcal{Q}_{N} f=\sum_{n=1}^{N}\left\langle f, \varphi_{n}\right\rangle \varphi_{n}
$$

\subsection{The stable sampling rate for the $\mathrm{ONB}+1$ frame}

A problem of interest is that where the samples are inner products with respect to the orthonormal basis $\left\{\varphi_{m}\right\}_{m \in \mathbb{N}}$. That is,

$$
\ell_{m, M}(f)=\ell_{m}(f)=\left\langle f, \varphi_{m}\right\rangle, \quad m=1, \ldots, M, M \in \mathbb{N} .
$$

For instance, these are Fourier coefficients if $\left\{\varphi_{m}\right\}_{m \in \mathbb{N}}$ is the Fourier basis, and hence the goal would be to compute a better approximation in the frame $\Phi$ from the given Fourier data. Note that this is an instance of the framework of $\S 4$ with $\mathrm{G}=\mathrm{H},\|\cdot\|\|=\| \cdot \|$ and $A^{\prime}=B^{\prime}=1$. Note also that $\|g\|_{M}=\left\|\mathcal{Q}_{M} g\right\|$. Recalling Proposition 4.6, we note that it suffices to estimate

$$
\sqrt{A_{M, N}^{\prime}}=\inf _{\substack{g \in \mathrm{H}_{N} \\\|g\|=1}}\left\|\mathcal{Q}_{M} g\right\| \geq 1-\sup _{\substack{g \in \mathrm{H}_{N} \\\|g\|=1}}\left\|g-\mathcal{Q}_{M} g\right\|,
$$

where $\mathrm{H}_{N}=\operatorname{span}\left\{g, \varphi_{1}, \ldots, \varphi_{N-1}\right\}$.

Lemma 5.1. For $M \geq N$, we have

$$
\sup _{\substack{g \in \mathrm{H}_{N} \\\|g\|=1}}\left\|g-\mathcal{Q}_{M} g\right\| \leq \frac{\left\|\psi-\mathcal{Q}_{M} \psi\right\|}{\left\|\psi-\mathcal{Q}_{N-1} \psi\right\|}
$$

Proof. Let $g \in \mathrm{H}_{N}$ and write $g=x_{0} \psi+\sum_{n=1}^{N-1} x_{n} \varphi_{n}$. Then

$$
\left\langle g, \varphi_{n}\right\rangle=x_{0}\left\langle\psi, \varphi_{n}\right\rangle+x_{n}, \quad n=1, \ldots, N-1 .
$$

Therefore

$$
g=x_{0} \psi+\sum_{n=1}^{N-1}\left(\left\langle g, \varphi_{n}\right\rangle-x_{0}\left\langle\psi, \varphi_{n}\right\rangle\right) \varphi_{n}=x_{0}\left(\psi-\mathcal{Q}_{N-1} \psi\right)+\mathcal{Q}_{N-1} g
$$

Rearranging gives

$$
g-\mathcal{Q}_{N-1} g=x_{0}\left(\psi-\mathcal{Q}_{N-1} \psi\right),
$$

and taking the norm of both sides, we find that

$$
\left|x_{0}\right|=\frac{\left\|g-\mathcal{Q}_{N-1} g\right\|}{\left\|\psi-\mathcal{Q}_{N-1} \psi\right\|} \leq \frac{\|g\|}{\left\|\psi-\mathcal{Q}_{N-1} \psi\right\|}=\frac{1}{\left\|\psi-\mathcal{Q}_{N-1} \psi\right\|} .
$$

Also, we have

$$
\left\langle g, \varphi_{n}\right\rangle=x_{0}\left\langle\psi, \varphi_{n}\right\rangle, \quad n \geq N
$$

Therefore

$$
\left\|g-\mathcal{Q}_{M} g\right\|=\left|x_{0}\right|\left\|\psi-\mathcal{Q}_{M} \psi\right\| .
$$

Combining this with the bound for $\left|x_{0}\right|$ gives the result. 
This leads us to the following result:

Theorem 5.2. Suppose that $\psi$ is such that $\left|\left\langle\psi, \varphi_{n}\right\rangle\right| \asymp n^{-\alpha}$ as $n \rightarrow \infty$ for some $\alpha>1 / 2$. Then the stable sampling rate

$$
\Theta^{\epsilon}(N, \theta) \leq C N,
$$

for some constant $C>0$ depending on $\alpha$ and $\theta$ only. Conversely, if $\left|\left\langle\psi, \varphi_{n}\right\rangle\right| \asymp \rho^{-n}$ as $n \rightarrow \infty$ for some $\rho>1$ then

$$
\Theta^{\epsilon}(N, \theta) \leq N+C
$$

where $C>0$ depends on $\alpha$ and $\theta$ only.

Proof. In the first case, the condition on the coefficients gives

$$
\left\|\psi-\mathcal{Q}_{N} \psi\right\|^{2}=\sum_{n \geq N}\left|\left\langle\psi, \varphi_{n}\right\rangle\right|^{2} \asymp N^{1-2 \alpha}, \quad N \rightarrow \infty .
$$

Hence Lemma 5.1 and the bound (5.2) give

$$
\sqrt{A_{M, N}^{\prime}} \geq 1-C_{\alpha} \frac{M^{1 / 2-\alpha}}{N^{1 / 2-\alpha}}
$$

In particular, $\sqrt{A_{M, N}^{\prime}} \gtrsim 1$ if $M \gtrsim N$. Recalling that $B^{\prime}=A^{\prime}=A=1$ and $B=2$ for this frame and using Proposition 4.6 gives the first result. For the second result, we notice that

$$
\left\|\psi-\mathcal{Q}_{N} \psi\right\|^{2} \asymp \frac{\rho^{-N}}{1-\rho} .
$$

We now argue as in the previous case.

This result shows that the stable sampling rate is linear when $\psi$ has algebraically or exponentiallydecaying coefficients in the orthonormal basis $\left\{\varphi_{n}\right\}_{n \in \mathbb{N}}$. Furthermore, the better $\psi$ is approximated in this basis, the smaller the stable sampling rate is, as evidenced by the case of exponentiallydecaying coefficients. In fact, Lemma 5.1 demonstrates the connection between the stable sampling rate and how well approximated $\psi$ is in the orthonormal basis $\left\{\varphi_{n}\right\}_{n \in \mathbb{N}}$. Specifically, the faster the projection errors $\left\|\psi-\mathcal{Q}_{M} \psi\right\|$ decay, the smaller $M \geq N$ needs to be so that $\frac{\left\|\psi-\mathcal{Q}_{M} \psi\right\|}{\left\|\psi-\mathcal{Q}_{N-1} \psi\right\|} \leq \delta$ for constant $0<\delta<1$. This is intuitive. The better $\psi$ is approximated by this basis, the more information the data, i.e. inner products with the $\varphi_{n}$, carries about the element $g$.

On the other hand, the worse $g$ is approximated the higher the stable sampling rate. Indeed, if $\left\|\psi-\mathcal{Q}_{M} \psi\right\| \asymp(\log (N))^{-1}$ then it is a simple exercise to show that the stable sampling rate is algebraic in $N$ with the power depending on $\theta$, i.e. $\Theta^{\epsilon}(N, \theta)=\mathcal{O}\left(N^{h(\theta)}\right)$ for some function $h(\theta) \geq 1$ with $h(\theta) \rightarrow \infty$ as $\theta \rightarrow 1^{+}$.

Remark 5.3 One can also determine a bound on the stable sampling rate for the problem of $\S 3$, where the data is the inner products

$$
\langle f, \psi\rangle, \quad\left\langle f, \psi_{m}\right\rangle, \quad m=1, \ldots, M-1 .
$$

Indeed, observe that

$$
A_{M, N}=\inf _{\substack{g \in \mathrm{H}_{N} \\\|g\|=1}} \sqrt{|\langle g, \psi\rangle|^{2}+\left\|\mathcal{Q}_{M-1} g\right\|^{2}} \geq A_{M-1, N}^{\prime},
$$

where $A_{M-1, N}^{\prime}$ is as in (5.2) with $M$ replaced by $M-1$. Hence the stable sampling rate for this problem satisfies the same bounds as those of Theorem 5.2. 


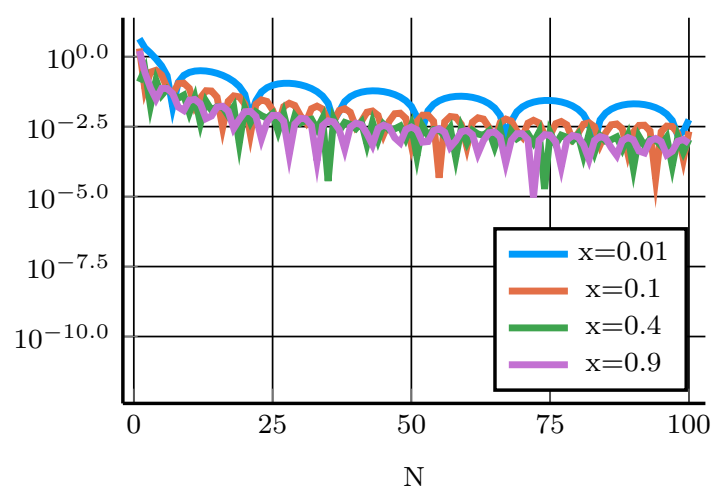

(a) Normalized Legendre polynomials

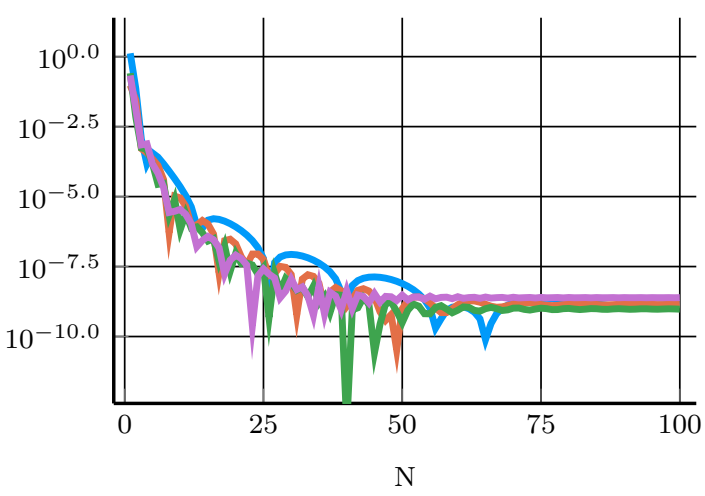

(b) ONB+1: Legendre polynomials $+\log x$

Figure 1: Pointwise error as a function of the polynomial degree $N$ for the approximation of a logarithmically singular function on $[0,1]$ using Legendre polynomials (left panel) and Legendre polynomials augmented with $\log x$ (right panel). The error is shown in four points in the interval $[0,1]$. In both cases, the approximation problem is solved using generalized sampling (5.1) with $M=2 N$. The generalized samples $\left\langle f, \phi_{m}\right\rangle_{m=1}^{M}$ were evaluated using adaptive numerical integration. The regularization threshold is $\epsilon=2 e^{-13}$.

\subsection{The approximation of functions with logarithmic singularities}

Let $\mathrm{H}=L^{2}(0,1)$. The scaled Legendre polynomials, $\varphi_{n}(x)=\sqrt{2 n+1} P_{n}(2 x-1)$, form an orthonormal basis for H. Here $P_{n}(x)$ is the usual Legendre polynomial, with normalization $P_{n}(1)=1$. This basis is extremely good at approximating smooth functions. However, many functions that may arise in applications, such as Green's functions or solutions to PDEs on domains with corners, fail to be smooth at a point $x$, yet posses a known type of singularity there. That is, in these applications we may want to approximate functions of the form

$$
f(x)=g(x)+w(x) h(x), \quad x \in(0,1)
$$

where $g, h$ are smooth functions, and $w \in L^{2}(0,1)$ is a known function which may be singular at, say, $x=0$. Such functions cannot generally be accurately approximated using polynomials alone. However, they can be more accurately capturing by enriching the polynomial basis with the function $w$. This gives a frame

$$
\Phi=\left\{\varphi_{n}\right\}_{n=0}^{\infty} \cup\{w\},
$$

for H. Indeed, since the $\varphi_{n}$ are an orthonormal basis, it quickly follows that

$$
\|f\|_{2}^{2}=\sum_{n=0}^{\infty}\left|\left\langle f, \varphi_{n}\right\rangle\right|^{2} \leq \sum_{n=0}^{\infty}\left|\left\langle f, \varphi_{n}\right\rangle\right|^{2}+|\langle f, w\rangle|^{2} \leq\|f\|^{2}+\|f\|^{2}\|w\|^{2} .
$$

Hence this is a frame with bounds $A \geq 1$ and $B \leq 1+\|w\|_{2}^{2}$.

The case of a logarithmic singularity, i.e. $w(x)=\log (x)$, is an important instance of the problem. Fig. 1 gives an illustration of the benefits of this frame over just the polynomial basis for approximating the simple yet singular function

$$
f(x)=e^{x}+\log (x) \cos (x) .
$$

The polynomial interpolation to $f$ converges poorly, as expected. However, adding just the single element $w(x)=\log (x)$ to the basis results in significantly faster convergence rates, shown in Fig. 
1(b). Importantly, note that the approximation scheme does not evaluate the smooth parts of $f$ separately. They are implicitly approximated simultaneously when approximating $f$ from its samples. Indeed, if the smooth parts of $f$ were known separately in an application, the approximation problem simplifies and there would be no need to construct a frame. Note also that the evaluation of the generalized samples (5.1) requires the evaluation of integrals, and this step is computationally demanding because the integrals are weakly singular. In subsequent examples, we shall consider a fully discrete approximation based on function samples.

Using Theorem 5.2, we may estimate the stable sampling rate for this problem:

Proposition 5.4. Let $\mathrm{H}=L^{2}(0,1), w(x)=\log (x), \varphi_{n}$ be the Legendre basis on $\mathrm{H}$, $\Phi$ be as in (5.6) and consider the sampling functionals (5.1). Then the stable sampling rate for this problem is linear in $N$, and specifically,

$$
\Theta(N, \theta) \leq \sqrt{\frac{\theta}{\theta-1}} N+1, \quad \forall \theta>1, N \in \mathbb{N} .
$$

Proof. The Legendre polynomials satisfy (see [6])

$$
\int_{0}^{1} P_{n}(2 x-1) \log (x) \mathrm{d} x=\frac{(-1)^{n+1}}{n(n+1)}, \quad n \geq 1 .
$$

Let $\psi(x)=\log (x)$. Then

$$
\left\|\psi-\mathcal{Q}_{M} \psi\right\|^{2}=\sum_{m \geq M}\left|\left\langle\psi, \varphi_{m}\right\rangle\right|^{2}=\sum_{m \geq M} \frac{2 m+1}{m^{2}(m+1)^{2}} \leq 2 \sum_{m \geq M} \frac{1}{m^{3}} \leq \frac{2}{(M-1)^{2}},
$$

and

$$
\left\|\psi-\mathcal{Q}_{M} \psi\right\|^{2} \geq 2 \sum_{m \geq M} \frac{1}{(m+1)^{3}} \geq \frac{2}{(M+1)^{2}} .
$$

Theorem 5.2 now gives that the stable sampling rate is linear in $N$. Moreover, Lemma 5.1 gives

$$
\sup _{\substack{g \in \mathrm{H}_{N} \\\|g\|=1}}\left\|g-\mathcal{Q}_{M} g\right\| \leq \frac{N^{2}}{(M-1)^{2}}
$$

Therefore

$$
\sup _{\substack{g \in \mathrm{H}_{N} \\\|g\|=1}}\left\|g-\mathcal{Q}_{M} g\right\| \leq 1-1 / \theta
$$

provided

$$
\frac{N^{2}}{(M-1)^{2}} \leq 1-1 / \theta \quad \Leftrightarrow \quad M \geq \sqrt{\frac{\theta}{\theta-1}} N+1
$$

as required.

\section{$5.3 \quad \mathrm{ONB}+K$ frames}

Functions with logarithmic singularities can be more acccurately approximately using the frame (5.4) than the Legendre polynomial basis alone. However, the accuracy may be limited, due to the presence of weak logarithmic singularities. To increase the accuracy one may consider a frame of the type

$$
\Phi=\left\{\varphi_{n}\right\}_{n=0}^{\infty} \cup\left\{\psi_{k}\right\}_{k=0}^{K-1},
$$

for fixed $K \geq 1$, where $\psi_{k}(x)=w(x) \varphi_{k}(x)$. 


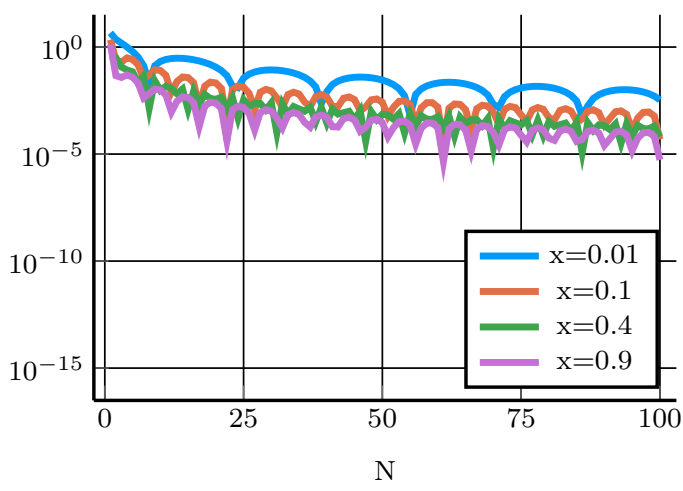

(a) Chebyshev interpolant

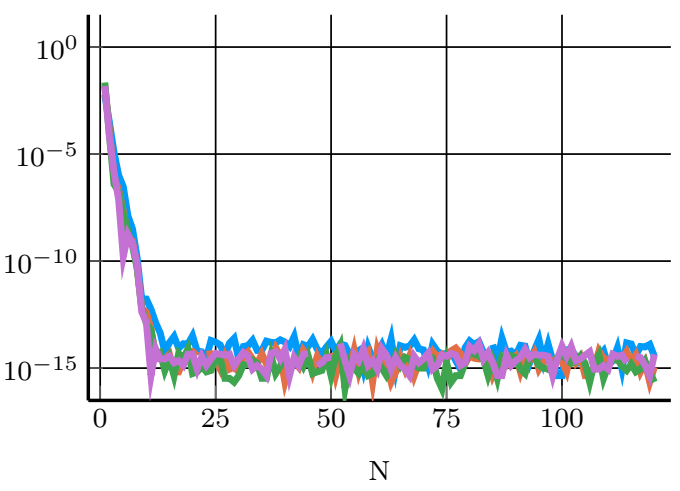

(b) Legendre $\mathrm{ONB}+K$ frame with $K=5$

Figure 2: Pointwise error as a function of the polynomial degree $N$ for the approximation of the logarithmically singular function (5.5) on [0,1], using Chebyshev interpolation (left panel) and the ONB $+K$ frame $\Phi$ with Legendre polynomials, $K=5$ and $w(x)=\log x$ (right panel). The error is shown in four points in the interval $[0,1]$. In both cases, the samples are function evaluations in the Chebyshev nodes. The left panel is based on interpolation, the right panel corresponds to a discrete least squares approximation with $M=2 N$ and regularization threshold $\epsilon=2 e^{-13}$.

Proposition 5.5. Let $\varphi_{n}$ be Legendre basis on $\mathrm{H}$ and $w \in L^{2}(0,1)$. Then (5.6) is a frame for any fixed $K \geq 1$, with frame bounds

$$
1 \leq A \leq B \leq 1+2 K^{2}
$$

Proof. First observe that $\psi_{k} \in L^{2}(0,1)$ since $w \in L^{2}(0,1)$ and $\varphi_{k} \in L^{\infty}(0,1)$. Second, we have

$$
\|f\|^{2}=\sum_{n=0}^{\infty}\left|\left\langle f, \varphi_{n}\right\rangle\right|^{2} \leq \sum_{n=0}^{\infty}\left|\left\langle f, \varphi_{n}\right\rangle\right|^{2}+\sum_{k=0}^{K-1}\left|\left\langle f, \psi_{k}\right\rangle\right|^{2},
$$

and therefore the lower frame condition holds with $A \geq 1$. Moreover,

$$
\begin{aligned}
\sum_{n=0}^{\infty}\left|\left\langle f, \varphi_{n}\right\rangle\right|^{2}+\sum_{k=0}^{K-1}\left|\left\langle f, \psi_{k}\right\rangle\right|^{2} & \leq\|f\|^{2}+\|f\|^{2}\|w\|_{L^{2}}^{2} \sum_{k=0}^{K-1}\left\|\varphi_{k}\right\|_{L^{\infty}}^{2} \\
& =\|f\|^{2}\left(1+2 \sum_{k=0}^{K-1}(2 k+1)\right)=\|f\|^{2}\left(1+2 K^{2}\right) .
\end{aligned}
$$

Here, in the penultimate step, we use the fact that $\left|\varphi_{n}(x)\right| \leq \varphi_{n}(1)=\sqrt{2 n+1}$ for $0 \leq x \leq 1$. This completes the proof.

In Fig. 2 we demonstrate the benefits of this frame for approximating the singular function given by (5.5). Here, rather than the inner products (5.1), we take the sampling functionals to be pointwise evaluations at the Chebyshev nodes on $[0,1]$, i.e.

$$
\ell_{m, M}(f)=f\left(x_{m, M}\right), \quad x_{m, M}=\frac{\cos \left(\frac{2 m-1}{2 M} \pi\right)+1}{2}, \quad m=1, \ldots, M .
$$

We also compare these approximations with the Chebyshev polynomial interpolant at these nodes. As is evident, Chebyshev interpolation performs poorly. Conversely, the convergence for the 


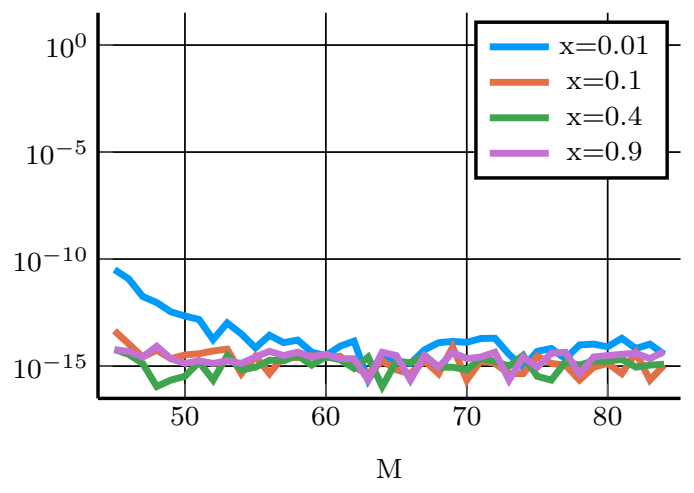

(a) Legendre points

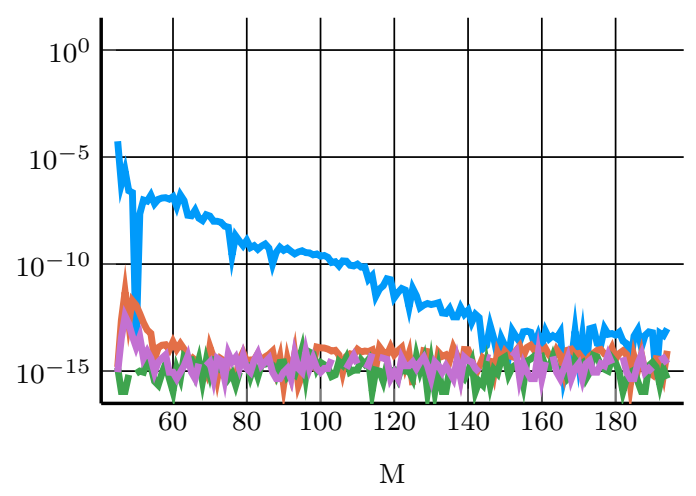

(b) Equispaced points

Figure 3: Pointwise error as a function of the number of samples $M$ for the ONB $+K$ frame based on Legendre polynomials, with degree $N=40, K=5$ and $w(x)=\log x$ as in Fig. 2(b). Similar to regular polynomial approximation, using Legendre points yields better accuracy than using equispaced points. They also require less oversampling, i.e., smaller values of $M$, to achieve the best error.

$\mathrm{ONB}+K$ frame is significantly faster. Fig. 2 also illustrates the stability of the numerical approximation using oversampling with $M=2 N$. The accuracy reaches machine precision, in spite of it requiring the solution of an extremely ill-conditioned linear system of equations, and this high level of accuracy is maintained as $N$ grows.

The influence of the oversampling factor $M$ is illustrated in Fig. 3. Here, the error is shown as a function of $M$, for constant $N=40$. Best accuracy is only achieved for $M>N$, i.e., when using some amount of oversampling. We have used discrete sampling in this figure using Legendre points (left panel) and equispaced points on $[0,1]$ (right panel). It is not unexpected that Legendre points are a better choice: less oversampling is needed to achieve the best accuracy for the given $N$.

The behaviour shown in Fig. 3 can be explained by computing the corresponding constants $\kappa_{M, N}^{\epsilon}$ and $\lambda_{M, N}^{\epsilon}$. This also serves to illustrate the stable sampling rate for this problem. Their values are shown in Fig. 4 for several choices of the oversampling factor $\gamma$, with $M$ and $N$ such that $M=\gamma N$. The convergence of both values to constants of modest size, in particular much smaller than $1 / \epsilon$, suggests that the stable sampling rate is indeed linear when sampling in the Legendre points. For equispaced points, as could be expected, this does not seem to be the case.

The results in Fig. 4 correspond to the threshold $\epsilon=1 e^{-5}$. For comparison, the experiment is repeated in Fig. 5 for the smaller threshold $\epsilon=1 e^{-8}$. The latter figure illustrates the larger upper bound of (4.21), on the order of $1 / \epsilon$, in the pre-asymptotic regime. Still, for the case of Legendre nodes, linear oversampling is sufficient to reach the $\epsilon$-independent (and small) limit (4.22).

The constants were computed following the approach described in Remark 4.9. We have run this experiment in higher precision arithmetic, in order to exclude the possibility of inaccuracies in their computation. An exponentially converging composite hp-graded quadrature rule was used to approximate the singular integrals that arise in the elements of the Gram matrix. Furthermore, in this case we also weighted the discrete samples in Legendre points by the square roots of the corresponding Gauss-Legendre quadrature weights: this discrete normalization ensures that $A^{\prime}=$ $B^{\prime}=1$ in (4.3) and leads to slightly smaller values of the constants (and, correspondingly, smaller error in the approximation). 


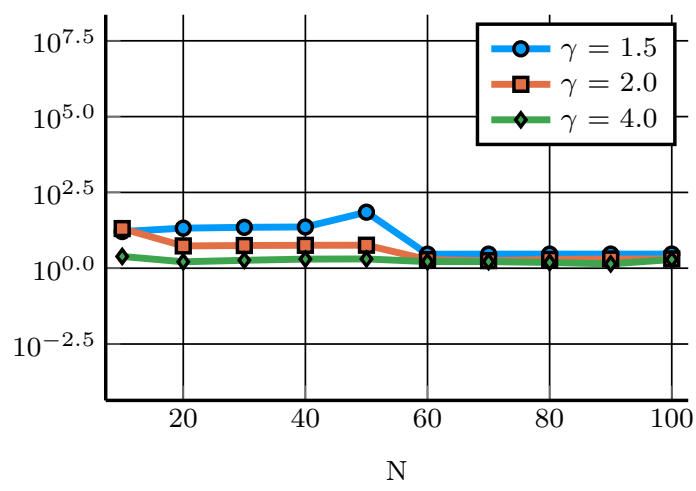

(a) $\kappa_{M, N}^{\epsilon}$, Legendre points

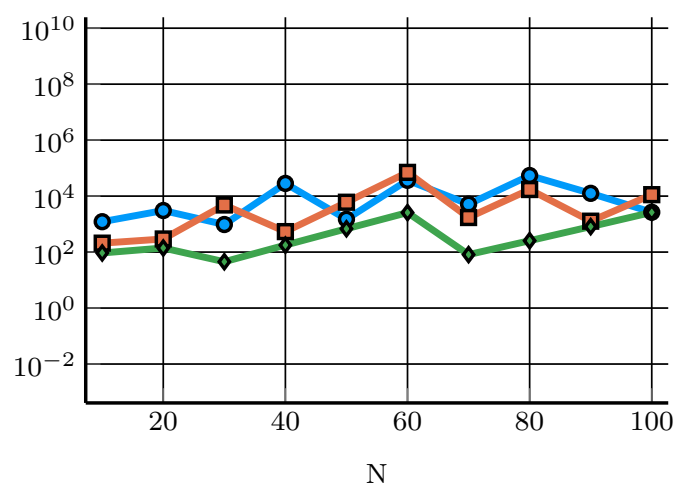

(c) $\kappa_{M, N}^{\epsilon}$, equispaced points

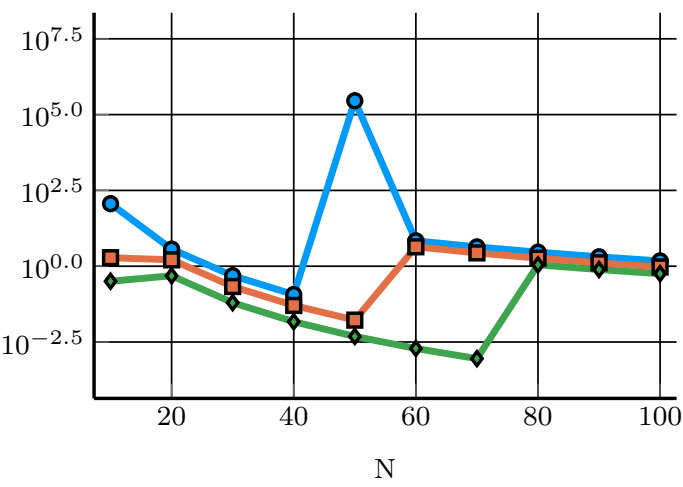

(b) $\lambda_{M, N}^{\epsilon}$, Legendre points

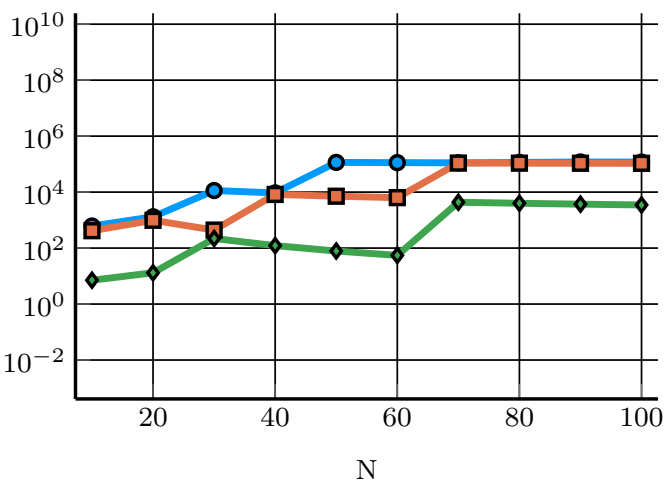

(d) $\lambda_{M, N}^{\epsilon}$, equispaced points

Figure 4: The values of $\kappa_{M, N}^{\epsilon}$ and $\lambda_{M, N}^{\epsilon}$ are shown as a function of $N$ with constant oversampling $M=\gamma N$ and varying factors $\gamma$. Legendre points (top row) and equispaced points (bottom row) are used for the $\mathrm{ONB}+K$ frame using Legendre polynomials, $w(x)=\log (x)$ and $K=5$. The threshold used here is $\epsilon=1 e^{-5}$ : the values are bounded for Legendre points but they approach $1 / \epsilon$ for equispaced points. Equispaced points require more than linear oversampling.

\section{Acknowledgements}

A preliminary version of this work was presented during the Research Cluster on "Computational Challenges in Sparse and Redundant Representations" at ICERM in November 2014. The authors would like to thank all the participants for the useful discussions and feedback received during the programme. The first author would also like to thank Juan Manuel Cárdenas and Sebastián Scheuermann. The first author is supported by NSERC grant 611675 , as well as an Alfred P. Sloan Research Fellowship The second author is supported by FWO-Flanders projects G.0641.11, G.A004.14 and by KU Leuven project C14/15/055.

\section{References}

[1] B. Adcock and A. C. Hansen. A generalized sampling theorem for stable reconstructions in arbitrary bases. J. Fourier Anal. Appl., 18(4):685-716, 2012. 


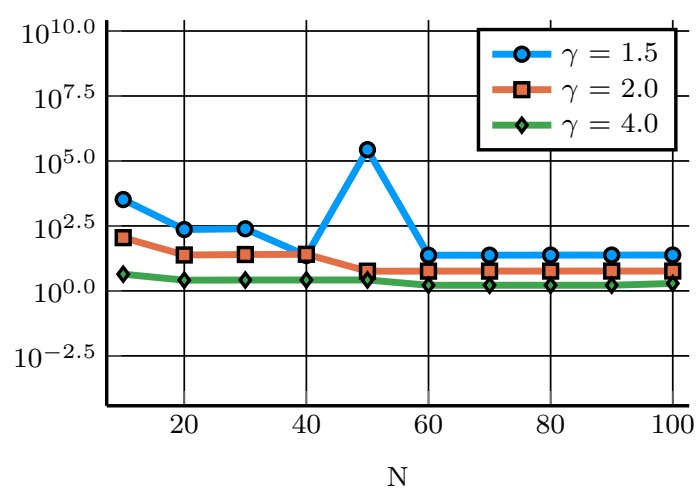

(a) $\kappa_{M, N}^{\epsilon}$, Legendre points

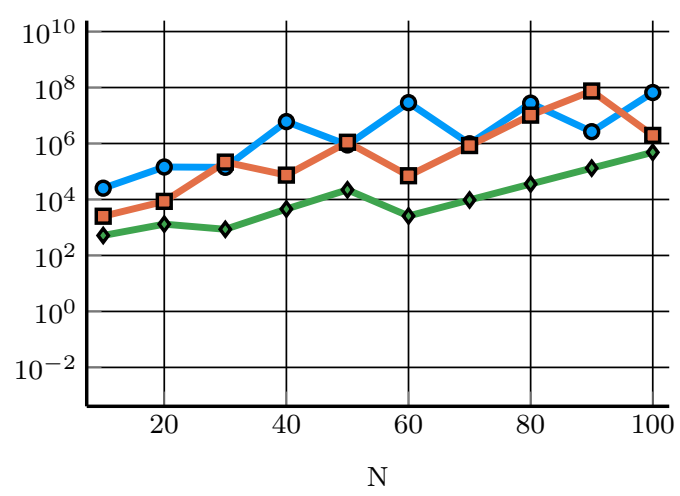

(c) $\kappa_{M, N}^{\epsilon}$, equispaced points

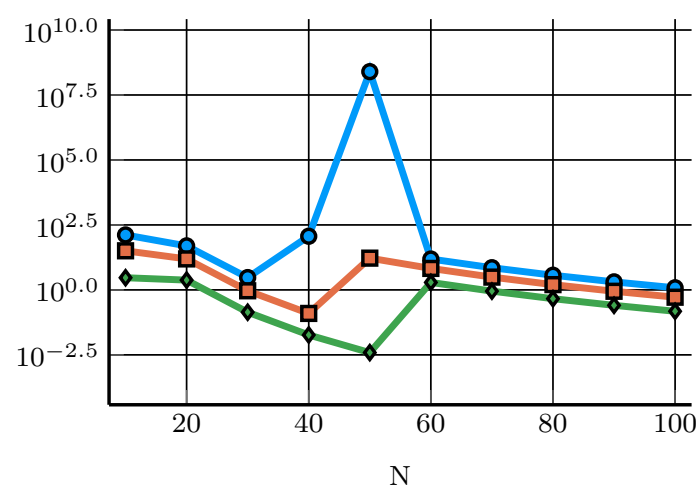

(b) $\lambda_{M, N}^{\epsilon}$, Legendre points

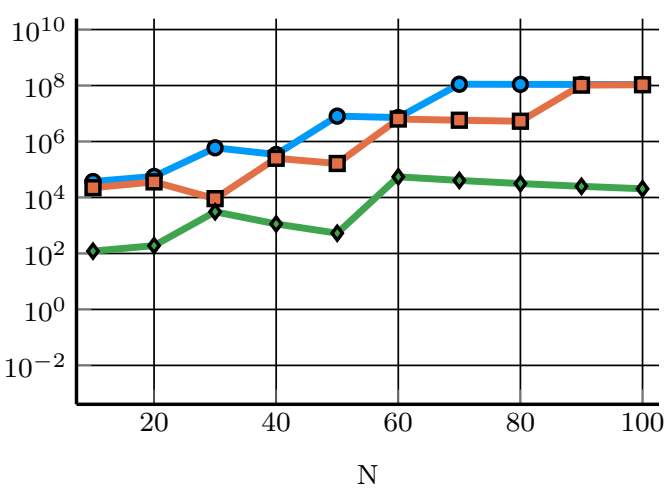

(d) $\lambda_{M, N}^{\epsilon}$, equispaced points

Figure 5: The same as Fig. 4 but with threshold $\epsilon=1 e^{-8}$. For Legendre nodes (top row), the intermediate peaks are higher, corresponding to the larger upper bounds in (4.21). However, the values settle down for increasing $N$, with a clear benefit for larger oversampling factors. This is in agreement with the limit (4.22). For equispaced samples, linear oversampling is not sufficient and, as in the previous figure, the values seem to approach $1 / \epsilon$. This again corresponds to (4.21).

[2] B. Adcock and A. C. Hansen. Stable reconstructions in Hilbert spaces and the resolution of the Gibbs phenomenon. Appl. Comput. Harmon. Anal., 32(3):357-388, 2012.

[3] B. Adcock, A. C. Hansen, and C. Poon. Beyond consistent reconstructions: optimality and sharp bounds for generalized sampling, and application to the uniform resampling problem. SIAM J. Math. Anal., 45(5):3114-3131, 2013.

[4] B. Adcock and D. Huybrechs. Frames and numerical approximation. SIAM Rev. (to appear), 2018.

[5] B. Adcock, D. Huybrechs, and J. Martín-Vaquero. On the numerical stability of Fourier extensions. Found. Comput. Math., 14(4):635-687, 2014.

[6] J. L. Blue. A Legendre polynomial integral. Math. Comp., 33(146):739-741, 1979.

[7] D. Boffi, N. Cavallini, and L. Gastaldi. The finite element immersed boundary method with distributed Lagrange multiplier. SIAM J. Numer. Anal., 53(6):2584-2604, 2015.

[8] O. Christensen. An Introduction to Frames and Riesz Bases. Applied and Numerical Harmonic Analysis. Birkhäuser, 2nd edition, 2016. 
[9] G. H. Golub and C. F. van Loan. Matrix computations. Johns Hopkins University Press, Baltimore, 3rd edition, 1996.

[10] A. C. Hansen. On the solvability complexity index, the n-pseudospectrum and approximations of spectra of operators. J. Amer. Math. Soc., 24(1):81-124, 2011.

[11] E. Heinemeyer, M. Lindner, and R. Potthast. Convergence and numerics of a multisection method for scattering by three-dimensional rough surfaces. SIAM J. Numer. Anal., 46(4):1780-1798, 2008.

[12] D. Huybrechs. On the Fourier extension of non-periodic functions. SIAM J. Numer. Anal., 47(6):4326$4355,2010$.

[13] F. Kasolis, E. Wadbro, and M. Berggren. Analysis of fictitious domain approximations of hard scatterers. SIAM J. Numer. Anal., 2015(5):2347-2362, 2015.

[14] M. Lindner. Infinite Matrices and their Finite Sections. Frontiers in Mathematics. Birkhäuser Verlag, Basel, 2006.

[15] M. Lyon. A fast algorithm for Fourier continuation. SIAM J. Sci. Comput., 33(6):3241-3260, 2012.

[16] R. Matthysen and D. Huybrechs. Fast algorithms for the computation of Fourier extensions of arbitrary length. SIAM J. Sci. Comput., 38(2):A899-A922, 2016.

[17] R. Matthysen and D. Huybrechs. Function approximation on arbitrary domains using Fourier extension frames. arXiv:1706.04848, 2017.

[18] D. Shirokoff and J.-C. Nave. A sharp-interface active penalty method for the incompressible NavierStokes equations. J. Sci. Comput., 62(1):53-77, 2015. 